\title{
EFFICIENT TESTS UNDER A WEAK CONVERGENCE ASSUMPTION
}

\begin{abstract}
BY ULRICH K. MÜLLER ${ }^{1}$
The asymptotic validity of tests is usually established by making appropriate primitive assumptions, which imply the weak convergence of a specific function of the data, and an appeal to the continuous mapping theorem. This paper, instead, takes the weak convergence of some function of the data to a limiting random element as the starting point and studies efficiency in the class of tests that remain asymptotically valid for all models that induce the same weak limit. It is found that efficient tests in this class are simply given by efficient tests in the limiting problem-that is, with the limiting random element assumed observed-evaluated at sample analogues. Efficient tests in the limiting problem are usually straightforward to derive, even in nonstandard testing problems. What is more, their evaluation at sample analogues typically yields tests that coincide with suitably robustified versions of optimal tests in canonical parametric versions of the model. This paper thus establishes an alternative and broader sense of asymptotic efficiency for many previously derived tests in econometrics, such as tests for unit roots, parameter stability tests, and tests about regression coefficients under weak instruments.
\end{abstract}

KEYWORDS: Robustness, unit root test, semiparametric efficiency.

\section{INTRODUCTION}

A CONTINUED FOCUS of the recent econometrics literature has been the development of asymptotically optimal inference procedures for nonstandard problems. The usual derivation proceeds in two steps: first, derive the optimal test in some canonical version of the model, usually assuming independent and identically distributed (i.i.d.) Gaussian disturbances. Second, derive a corresponding robustified test that yields the same asymptotic rejection probability under the null hypothesis (and local alternatives) for a wide range of data generating processes. This second step is important, because in most applications, one would not want to assume that the canonical model is necessarily correct. Examples of this approach include the univariate unit root tests by Elliott, Rothenberg, and Stock (1996) (subsequently abbreviated ERS), Elliott (1999), Müller and Elliott (2003), and Müller (2009); the unit root test with stationary covariates by Elliott and Jansson (2003); the test of the null hypothesis of no cointegration with known cointegrating vector by Elliott, Jansson, and Pesavento (2005); the test of the null hypothesis of cointegration by Jansson (2005); the parameter stability tests by Nyblom (1989), Andrews and Ploberger (1994), and Elliott and Müller (2006); the tests about regression coefficients

\footnotetext{
${ }^{1}$ A previous version of this paper was circulated under the title "An Alternative Sense of Asymptotic Efficiency." The author would like to thank Whitney Newey, three anonymous referees, Mark Watson, seminar participants at Berkeley, Brown, Harvard/MIT, Penn State, and Stanford, and conference participants at the NBER Summer Institute 2008 and the ESEM Summer Meeting 2008 for helpful comments and discussions, as well as Jia Li for excellent research assistance. Financial support by the NSF through Grant SES-0751056 is gratefully acknowledged.
} 
with nearly integrated regressors by Stock and Watson (1996) and Jansson and Moreira (2006); and the tests about regression coefficients with weak instruments by Andrews, Moreira, and Stock $(2006,2008)$. The robustness in the second step is established by making suitable primitive assumptions, so that the law of large numbers and (functional) central limit theorems can be invoked to a specific function of the data, and the large sample properties of the test then follow from the continuous mapping theorem. By construction, these tests are thus optimal in the canonical specification of the model and are asymptotically valid whenever the weak convergence implied by the limit theorems holds.

This paper, instead, starts out with a specific function $X_{T}=h_{T}\left(Y_{T}\right)$ of the data $Y_{T}$. This function is chosen so that typical primitive assumptions imply the weak convergence of $X_{T}$ to a random element $X$, whose distribution is different under the null and local alternative. For instance, in the unit root testing problem, a suitable transformation of the data to the unit interval converges weakly to a Wiener process under the null hypothesis, and to an OrnsteinUhlenbeck process under the local alternative. We then ask the question, "What is the efficient test in the class $\mathfrak{C}$ of tests that remain asymptotically valid whenever $X_{T}=h_{T}\left(Y_{T}\right)$ converges weakly to its null limiting distribution?"

It is clear that the performance of the robustified test derived from the canonical model provides an important benchmark, as it is a member of this class for an appropriately defined function $h_{T}$. But it is not clear that one could not do better. After all, if one knew that the data were generated by some model other than the canonical model, then the best test would typically not coincide with the best test in the canonical model. For instance, Rothenberg and Stock (1997) and Jansson (2008) derived unit root tests with higher local asymptotic power than ERS's test for the AR(1) model with nonnormal i.i.d. driving errors, which continue to induce the weak convergence to a Wiener and Ornstein-Uhlenbeck process.

The main contribution of the paper is a simple characterization of the efficient test in the class $\mathfrak{C}$, that is the best test among all tests that remain asymptotically valid whenever $X_{T}$ converges weakly to its null limiting distribution. The characterization is in terms of the efficient test in the "limiting problem," where the limiting random element $X$ is directly observed. For instance, in the unit root testing problem, the Radon-Nikodym derivative of the distribution of an Ornstein-Uhlenbeck process (for some fixed mean reversion parameter) and the distribution of a Wiener process is, by the Neyman-Pearson lemma, the point-optimal test statistic in the limiting problem. It is shown that the efficient test in the limiting problem $\varphi^{*}(X)$, evaluated at the small sample analogue $\varphi^{*}\left(X_{T}\right)=\varphi^{*}\left(h_{T}\left(Y_{T}\right)\right)$, is the efficient test in $\mathfrak{C}$. In the unit root testing case, $\varphi^{*}\left(X_{T}\right)$ is asymptotically equivalent to the test statistic derived by ERS, so that this result establishes a sense of efficiency of their test also outside the canonical model.

Whether or not a given test $\varphi_{T}\left(Y_{T}\right)$ is a member of the class $\mathfrak{C}$ is determined by its asymptotic rejection probability under data generating processes that induce $X_{T}$ to converge to its null limiting distribution. One could alternatively 
restrict attention to tests of a particular functional form, such as tests that can be written as continuous functions of $X_{T}, \varphi_{T}\left(Y_{T}\right)=\varphi_{c}\left(X_{T}\right)$ with $\varphi_{c}$ continuous. For instance, Stock (1999) considered tests of this form in the context of unit root testing. By the continuous mapping theorem, the asymptotic rejection probability of the test $\varphi_{c}\left(X_{T}\right)$ is determined by the distribution of $X$. Any test of the form $\varphi_{T}\left(Y_{T}\right)=\varphi_{c}\left(X_{T}\right)$ is, therefore, a member of $\mathfrak{C}$ if (and only if) $\varphi_{c}(X)$ is a valid test in the limiting problem. As a corollary, the main result thus implies that if $\varphi^{*}$ is continuous, $\varphi^{*}\left(X_{T}\right)$ is also best among all such tests. This corollary might be quite useful, since as a practical matter, tests of the form $\varphi_{c}\left(X_{T}\right)$ are easy to relate to and thus a reasonable starting point.

The efficiency of $\varphi^{*}\left(X_{T}\right)$ in the class $\mathfrak{C}$ is stronger though, as noncontinuous tests are not ruled out a priori. For instance, one might hope that a more powerful unit root test can be obtained by first carefully checking whether the data are drawn from an $\mathrm{AR}(1)$ with i.i.d. non-Gaussian disturbances. If they seem to, apply a Jansson (2008) type test, which is more powerful than ERS's statistic. If not, revert to ERS's statistic. The efficiency of $\varphi^{*}\left(X_{T}\right)$ in $\mathfrak{C}$ implies, however, that any such scheme cannot result in a test that is a member of $\mathfrak{C}$ : Whenever a test yields higher asymptotic power for some model that induces convergence to an Ornsetein-Uhlenbeck process, there also exists a data generating process for $Y_{T}$ where $X_{T}$ converges to a Wiener process, and the test overrejects asymptotically.

The value of the efficiency claim about $\varphi^{*}\left(X_{T}\right)$ depends, in general, on the appeal of restricting attention to tests in $\mathfrak{C}$. The class obviously depends on the choice of the function $h_{T}$. The strongest case can be made if the weak convergence assumption about $h_{T}\left(Y_{T}\right)$ embeds all relevant knowledge about the testing problem. In time series applications, primitive regularity assumptions (such as mixing and moment assumptions) are arguably sometimes invoked only because they imply a certain weak convergence, but not because there are particularly good reasons for the true model to satisfy these constraints. In such instances, it seems quite natural to restrict attention to tests that remain valid whenever the implied weak convergences hold. On the other hand, if, say, an economic model implies some observable data to follow an AR(1) with i.i.d. shocks, then it does not necessarily make sense to restrict attention to unit root tests that remain valid whenever the data suitably transformed converge to a Wiener process. In general, knowledge of the i.i.d. nature of disturbances (or observations in a cross sectional setting) is not easily reexpressed in terms of weak convergence for some $h_{T}\left(Y_{T}\right)$. In applications with such knowledge, the test $\varphi^{*}\left(X_{T}\right)$ might still be a reasonable starting point, but it only provides a fairly limited answer to the question of efficient inference.

One appeal of the framework of this paper is that the characterization of the efficient test is quite straightforward, even for nonstandard testing problems. The basic result that efficient tests in the limiting problem translate to efficient tests in $\mathfrak{C}$ holds quite generally, also for cases where tests are restricted to satisfy some asymptotic unbiasedness or conditional similarity constraint, or to be 
invariant. Standard arguments from testing theory can thus be applied to identify an efficient test in the limiting problem. The results of this paper may thus be seen as a formalization and generalization of Sowell (1996), who employed the Neyman-Pearson lemma to the limiting measure of partial sums of sample moment conditions to derive parameter stability tests in a generalized method of moments (GMM) framework. Additional potential applications include a broader sense of asymptotic efficiency of the test statistics derived in the 14 papers cited in the first paragraph of this Introduction. Finally, the results of this paper also imply efficiency of some recent nonstandard methods that take a weak convergence assumption as their starting point, such as those suggested in Müller and Watson (2008b, 2008a) and Ibragimov and Müller (2010).

The approach pursued in this paper is related to the literature on semiparametric efficiency and Le Cam's limits of experiments theory; see Le Cam (1986), Bickel, Klaassen, Ritov, and Wellner (1998), Newey (1990), van der Vaart (1998), and Shiryaev and Spokoiny (2000) for introductions. The starting point of the study of semiparametric efficient tests is a semiparametric model, which is a description of the distribution of $Y_{T}$ for each sample of size $T$ as a function of the parameter of interest, and an infinite dimensional nuisance parameter. Asymptotic efficiency results can then be deduced by limit of experiments arguments from the limiting behavior of the log-likelihood process. In most applications, the log-likelihood is locally asymptotically normal (see, for instance, Choi, Hall, and Schick (1996) for general results on semiparametric efficient tests), but especially time series applications also give rise to locally asymptotic quadratic behavior (as defined in Jeganathan (1995)). For instance, Ling and McAleer (2003) derived semiparametric efficient estimators for an autoregressive moving average model with generalized autoregressive conditional heteroskedasticity errors of unknown distribution, and Jansson (2008) derived semiparametric efficient unit root test in an AR(1) with i.i.d. disturbances of unknown distribution. In this literature, as well as in this paper, the heuristic argument is the same: if for any specific sequence of distributions of $Y_{T}$ under the local alternative, it is possible to identify a sequence of distributions under the null that also satisfies the constraints and for which the same test is the best test, then this test is the overall efficient test. The details of the construction are different, though, because in the traditional semiparametric setup, the constraints are on the small sample distribution of $Y_{T}$. In contrast, the restriction to tests in $\mathfrak{C}$ amounts to a constraint on the limiting behavior of the distributions of $Y_{T}$. Also, the limit experiment of a semiparametric problem explicitly characterizes the effect of not knowing the nonparametric component,${ }^{2}$ in contrast to the limiting problem of this paper. We explicitly compare the two constructions in a simple locally asymptotically normal (LAN) model in Section 2.5 below. As a practical matter, the approach of this paper leads to

\footnotetext{
${ }^{2} \mathrm{Or}$, at least, of not knowing the parameter of a suitably chosen parametric submodel as in Stein (1956).
} 
a relatively more easily applicable characterization of the efficient tests, especially for nonstandard problems, although arguably at the cost of providing a less definite answer.

The remainder of the paper is organized as follows. Section 2 introduces the formal framework and contains the main result. Section 3 discusses extensions regarding consistently estimable nuisance parameters, invariance restrictions, and uniformity issues. A running example throughout Sections 2 and 3 is the problem of testing for an autoregressive unit root in a univariate time series. Section 4 discusses in detail three applications: Elliott and Jansson's (2003) point-optimal tests for unit roots with stationary covariates; Andrews, Moreira, and Stock's (2006) optimal tests for linear instrumental variable regressions; and Sowell's (1996) tests for GMM parameter stability. Section 5 concludes. All proofs are collected in the Appendix.

\section{EFFICIENCY UNDER A WEAK CONVERGENCE ASSUMPTION}

\subsection{Setup}

The following notation and conventions are used throughout the paper: All limits are taken as $T \rightarrow \infty$. Suppose $S_{1}$ and $S_{2}$ are spaces with metrics $d_{S_{1}}$ and $d_{S_{2}}$. Then $\mathfrak{B}\left(S_{1}\right)$ denotes the Borel $\sigma$-algebra of $S_{1}$. Furthermore, if $\mu$ is a probability measure on $\mathfrak{B}\left(S_{1}\right)$, then its image measure under the $\mathfrak{B}\left(S_{1}\right) \backslash$ $\mathfrak{B}\left(S_{2}\right)$ measurable mapping $f: S_{1} \mapsto S_{2}$ is denoted $f \mu$. If no ambiguity arises, we suppress the dummy variable of integration, that is, we write $\int f d \mu$ for $\int f(x) d \mu(x)$. By default, the product space $S_{1} \times S_{2}$ is equipped with the metric $d_{S_{1}}+d_{S_{2}}$. We write $\mu_{T} \rightsquigarrow \mu_{0}$ or $X_{T} \rightsquigarrow X_{0}$ for the weak convergence of the random elements $X_{0}, X_{1}, \ldots$ with probability measures $\mu_{0}, \mu_{1}, \ldots$ on $\mathfrak{B}\left(S_{1}\right)$ and write $X_{T} \stackrel{p}{\rightarrow} X$ for convergence in probability. The $\mathbb{R} \mapsto \mathbb{R}$ function $x \mapsto$ $\lfloor x\rfloor$ is the integer part of $x$.

In a sample of size $T$, suppose we observe data $Y_{T} \in \mathbb{R}^{n T}$, which is the $T$ th row of a double array of $T$ random vectors of fixed dimension $n$. For given $T$, a statistical model for $Y_{T}$ with parameter $\theta$ in the metric space $\Theta$ is a family of probability measures $\Lambda_{T}=\left\{\lambda_{T}(\theta): \theta \in \Theta\right\}$ on $\mathfrak{B}\left(\mathbb{R}^{n T}\right)$, so that $\int_{A} d \lambda_{T}(\theta)$ is a $\mathfrak{B}(\Theta)$-measurable function of $\theta$ for each $A \in \mathfrak{B}\left(\mathbb{R}^{n T}\right)$. Let $m$ be a model for the whole sequence of observations $\left\{Y_{T}\right\}_{T=1}^{\infty}$ with the same parameter space $\Theta$ for all $T$, that is, $m=\left\{\Lambda_{T}\right\}_{T=1}^{\infty}$. We write $F_{T}(\theta, m)$ for the distribution of $Y_{T}$ in model $m$ with parameter value $\theta$. The hypotheses of interest are

$$
H_{0}: \theta \in \Theta_{0} \text { against } H_{1}: \theta \in \Theta_{1},
$$

where $\Theta_{0} \cup \Theta_{1}=\Theta$.

Let $h_{T}$ be a a given sequence of measurable functions $h_{T}: \mathbb{R}^{n T} \mapsto S$, where $S$ is a complete and separable metric space. Denote by $P_{T}(m, \theta)$ the distribution of $X_{T}=h_{T}\left(Y_{T}\right)$ in model $m$ with parameter $\theta$, that is, $P_{T}(m, \theta)=h_{T} F_{T}(m, \theta)$ 
in the notation defined above. Suppose the typical model $m$ induces the weak convergence

$$
P_{T}(m, \theta) \rightsquigarrow P(\theta) \quad \text { pointwise for all } \theta \in \Theta,
$$

where $\{P(\theta): \theta \in \Theta\}$ is a statistical model on $\mathfrak{B}(S)$. We assume throughout that the probability measures $P\left(\theta_{1}\right)$ and $P\left(\theta_{2}\right)$ on $\mathfrak{B}(S)$ are equivalent for all $\theta_{1}, \theta_{2} \in \Theta$, so that the family of measures $P(\theta)$ is dominated by $\mu_{P}=P\left(\theta_{0}\right)$ for some fixed $\theta_{0} \in \Theta$. The parameter $\theta$ should be thought of as describing local alternatives, such as the magnitude of the Pitman drift.

Unit Root TEST EXAMPLE: Consider testing for a unit root in a model with no deterministics against the local-to-unity alternative: We observe data $Y_{T}=\left(u_{T, 1}, \ldots, u_{T, T}\right)^{\prime}$ from the model $u_{T, t}=\rho_{T} u_{T, t-1}+\nu_{T, t}$ and $u_{T, 0}=0$ for all $T$, where $\rho_{T}=1-c / T$ for some fixed $c \geq 0$ and the hypotheses are $H_{0}: c=0$ against $H_{1}: c>0$ (so that $n=1, \theta=c, \Theta_{0}=\{0\}$, and $\Theta_{1}=(0, \infty)$ ). Let $\hat{\omega}_{T}^{2}$ be a specific, "reasonable" long-run variance estimator. With $S=D_{[0,1]}$ being the space of cadlag functions on the unit interval, equipped with the Billingsley (1968) metric, a typical model $m$ for the disturbances $\nu_{T, t}$ leads to $T^{-1 / 2} \hat{\omega}_{T}^{-1} u_{T,\lfloor\cdot T\rfloor}=h_{T}\left(Y_{T}\right)=\hat{J}_{T}(\cdot) \rightsquigarrow J_{c}(\cdot)$ on $D_{[0,1]}$, where $J_{c}$ is an OrnsteinUhlenbeck process $J_{c}(s)=\int_{0}^{s} e^{-c(s-r)} d W(r)$ with $W$ being a standard Wiener process. It is well known that the measure of $J_{c}$ is absolutely continuous with respect to the measure of $J_{0}=W$.

\subsection{Limiting Problem}

In the typical model $m$, the observed random element $X_{T}$ satisfies $X_{T} \rightsquigarrow$ $X \sim P(\theta)$. It will be useful in the sequel to first consider in detail the limiting problem, where $X$ is assumed to be observed:

$$
H_{0}^{l p}: X \sim P(\theta), \quad \theta \in \Theta_{0} \quad \text { against } \quad H_{1}^{l p}: X \sim P(\theta), \quad \theta \in \Theta_{1} .
$$

Possibly randomized tests of $H_{0}^{l p}$ are measurable functions $\varphi_{S}: S \mapsto[0,1]$, where $\varphi_{S}(x)$ indicates the probability of rejection conditional on observing $X=x$, so that the overall rejection probability of the test $\varphi_{S}$ is $\int \varphi_{S} d P(\theta)$ when $X \sim P(\theta)$. The test $\varphi_{S}$ is thus of level $\alpha$ if $\sup _{\theta \in \Theta_{0}} \int \varphi_{S} d P(\theta) \leq \alpha$. In many nonstandard problems, no uniformly most powerful test exists, so consider tests that maximize a weighted average power criterion

$$
\operatorname{WAP}\left(\varphi_{S}\right)=\int\left(\int \varphi_{S} d P(\theta)\right) d w(\theta),
$$

where $w$ is a probability measure on $\Theta_{1}$. In general, the weighting function $w$ describes the importance a researcher attaches to the ability of the test to reject for certain alternatives. A point-optimal test, as suggested by King (1988), 
is a special case of a weighted average power (WAP) maximizing test for a degenerate weighting function $w$ that puts all mass on one point. Also, if a uniformly most powerful test exists, then it maximizes WAP for all choices for $w$. The WAP criterion is statistically convenient, since by standard arguments, the WAP maximizing test equivalently maximizes power against the single alternative $H_{1, w}^{l p}: X \sim \int P(\theta) d w(\theta)$.

With the WAP criterion as the efficiency measure, efficient level- $\alpha$ tests $\varphi_{S}^{*}$ in the limiting problem (3) maximize WAP subject to $\sup _{\theta \in \Theta_{0}} \int \varphi_{S} d P(\theta) \leq \alpha$.

UNIT RoOT TEST EXAMPLE-CONTINUED: The weak convergence $\hat{J}_{T}(\cdot) \rightsquigarrow$ $J_{c}(\cdot)$ leads to the limiting problem where we observe the continuous time process $X$, and $H_{0}^{l p}: X \sim J_{0}(\cdot)$ against $H_{1}^{l p}: X \sim J_{c}(\cdot)$ with $c>0$. As a weighting function in the WAP criterion, consider a degenerate distribution with all mass at $c_{1}$, so that we consider a point-optimal test, just as ERS. By Girsanov's theorem, the Radon-Nikodym derivative of the distribution of $J_{c_{1}}$ with respect to the distribution of $J_{0}$, evaluated at $X$ is given by $L(X)=$ $\exp \left[-\frac{1}{2} c_{1}\left(X(1)^{2}-1\right)-\frac{1}{2} c_{1}^{2} \int_{0}^{1} X(s)^{2} d s\right]$. Thus, by the Neyman-Pearson lemma, the point-optimal test in the limiting problem is $\varphi_{S}^{*}(X)=\mathbf{1}[L(X)>\mathrm{cv}]$, where the critical value cv solves $P\left(L\left(J_{0}(\cdot)\right)>\mathrm{cv}\right)=\alpha$.

When $\Theta_{0}$ is not a singleton, that is, if $H_{0}^{l p}$ is composite, the derivation of a WAP maximizing test is often more involved. The weighted average power maximizing test under a composite null hypothesis is typically given by the Neyman-Pearson test of $H_{0, v}^{l p}: X \sim \int P(\theta) d v(\theta)$ against $H_{1, w}^{l p}: X \sim$ $\int P(\theta) d w(\theta)$, where $v$ is the least favorable distribution for $\theta$; see Chapter 3.8 of Lehmann (1986) for a discussion. For many problems, however, it is difficult to identify the least favorable distribution $v$ (see, for instance, King (1988), Sriananthakumar and King (2006), Müller and Watson (2008a), or Elliott and Müller (2009)). To make further progress, researchers often restrict the class of tests under consideration by additional constraints and derive the best test in the restricted class. Sometimes the WAP maximizing test in the restricted class turns out to be uniformly most powerful (that is, maximizes WAP for all weighting functions), so that the issue of how to choose an appropriate weighting function is also avoided by imposing additional constraints.

We discuss invariance as a restriction in Section 3.2 below and focus here on two other constraints on the tests $\varphi_{S}$. First, consider

$$
\int \varphi_{S} d P(\theta) \geq \pi_{0}(\theta) \text { for all } \theta \in \Theta_{1}
$$

for some function $\pi_{0}: \Theta_{1} \mapsto \mathbb{R}$. The formulation (5) allows for a range of cases: with $\pi_{0}=0$, (5) never binds; with $\pi_{0}=\alpha$, (5) imposes unbiasedness; with $\pi_{0}$ equal to the power of the locally best test for all $\theta$ close to $\Theta_{0}$, (5) effectively selects $\varphi_{S}$ to be the locally best test. 
Second, consider a (conditional) similarity constraint of the form

$$
\int\left(\varphi_{S}-\alpha\right) f_{S} d P(\theta)=0 \quad \text { for all } \theta \in \bar{\Theta}_{0} \text { and } f_{S} \in \mathcal{F}_{S}
$$

for some $\bar{\Theta}_{0} \subset \Theta_{0}$, which would typically be the intersection of $\Theta_{0}$ with the closure of $\Theta_{1}$, and $\mathcal{F}_{S}$ some set of $\mu_{P}$ almost everywhere continuous and bounded functions $f_{S}: S \mapsto \mathbb{R}$. With $\mathcal{F}_{S}$ only containing the zero function, (6) never binds. With $\mathcal{F}_{S}$ only containing the function that is equal to $1,(6)$ imposes similarity on $\bar{\Theta}_{0}$. Finally, suppose $\vartheta: S \mapsto U$ is a $\mu_{P}$ almost everywhere continuous function and that $\mathcal{F}_{S}$ contains all $S \mapsto \mathbb{R}$ functions of the form $f_{U} \circ \vartheta$, where $f_{U}: U \mapsto \mathbb{R}$ is continuous and bounded. Then (6) amounts to the restriction that the rejection probability of $\varphi_{S}$, conditional on $\vartheta(X)$, is equal to $\alpha$ for all $\theta \in \bar{\Theta}_{0}$, so that $\varphi_{S}$ is a conditionally similar test.

To sum up, we will refer to level- $\alpha$ tests $\varphi_{S}^{*}$ in the limiting problem (3) as efficient when $\varphi_{S}^{*}$ maximizes weighted average power (4), subject to (5) and (6).

\subsection{Class of Models and Tests}

In the original hypothesis testing problem (1) with $Y_{T}$ observed, tests are measurable functions $\varphi_{T}: \mathbb{R}^{n T} \mapsto[0,1]$, where $\varphi_{T}\left(y_{T}\right)$ indicates the probability of rejection conditional on observing $Y_{T}=y_{T}$. The overall rejection probability of the test $\varphi_{T}$ in model $m$ with parameter $\theta$ is thus given by $\int \varphi_{T} d F_{T}(m, \theta)$.

As in the discussion of the limiting problem, we consider weighted average power as the criterion to measure the efficiency of tests $\varphi_{T}$. In analogy to $\operatorname{WAP}\left(\varphi_{S}\right)$ in the limiting problem (4), define

$$
\operatorname{WAP}_{T}\left(\varphi_{T}, m\right)=\iint \varphi_{T} d F_{T}(m, \theta) d w(\theta) .
$$

Also, define the asymptotic null rejection probability $\left(\mathrm{ARP}_{0}\right)$ of the test $\varphi_{T}$ in model $m$ as

$$
\operatorname{ARP}_{0}\left(\varphi_{T}, m\right)=\sup _{\theta \in \Theta_{0}} \limsup _{T \rightarrow \infty} \int \varphi_{T} d F_{T}(m, \theta) .
$$

With these definitions, an asymptotically powerful level- $\alpha$ test $\varphi_{T}$ has large $\lim _{T \rightarrow \infty} \operatorname{WAP}_{T}\left(\varphi_{T}, m\right)$, while $\operatorname{ARP}_{0}\left(\varphi_{T}, m\right) \leq \alpha$. If the exact model $m$ is unknown, it makes sense to impose that $\operatorname{ARP}_{0}\left(\varphi_{T}, m\right) \leq \alpha$ for a large set of models $m$.

Now the key premise of this paper is that an interesting set of such models is the set $\mathcal{M}$ of models satisfying (2), that is,

$$
\mathcal{M}=\left\{m: P_{T}(m, \theta)=h_{T} F_{T}(m, \theta) \rightsquigarrow P(\theta) \text { for all } \theta \in \Theta\right\} .
$$


We thus restrict attention to the class $\mathfrak{C}$ of tests $\varphi_{T}$ with asymptotic null rejection probability no larger than the nominal level for all models $m \in \mathcal{M}$, that is, $\varphi_{T} \in \mathfrak{C}$ if and only if

$$
\sup _{\theta \in \Theta_{0}} \limsup _{T \rightarrow \infty} \int \varphi_{T} d F_{T}(m, \theta) \leq \alpha \quad \text { for all } m \in \mathcal{M} .
$$

The constraint (8) only imposes pointwise validity: for a given $m \in \mathcal{M}$ and $\theta \in$ $\Theta_{0}$, the test $\varphi_{T}$ has asymptotic rejection probability of at most $\alpha$. A stronger, uniform restriction analogous to (8) is discussed in Section 3.3 below. Also, the constraint (8) technically requires asymptotic validity only for models, parametrized by $\theta \in \Theta$, that satisfy (2). But for any sequence of measures $G_{T}$ on $\mathfrak{B}\left(\mathbb{R}^{n T}\right)$ with $h_{T} G_{T} \rightsquigarrow P\left(\theta_{G}\right)$ for some $\theta_{G} \in \Theta_{0}$, one can construct a model $m \in \mathcal{M}$ such that $F_{T}\left(m, \theta_{G}\right)=G_{T} \cdot{ }^{3}$ Members of $\mathfrak{C}$ thus have no more than nominal asymptotic rejection probability for all data generating processes for $Y_{T}$ that induce $X_{T}=h_{T}\left(Y_{T}\right)$ to converge weakly to some null limiting distribution $P(\theta), \theta \in \Theta_{0}$.

The class $\mathfrak{C}$ is never empty. Any test statistic of the form $\tau \circ h_{T}$, where $\tau: S \mapsto \mathbb{R}$ is a $\mu_{P}$ almost everywhere continuous function, has a limiting distribution equal to $\tau P(\theta)$ for any model $m \in \mathcal{M}$ and $\theta \in \Theta$ by virtue of (2) and the continuous mapping theorem. The class $\mathfrak{C}$ thus includes all tests $\varphi_{T}$ of the form $\mathbf{1}\left[\tau \circ h_{T}>\mathrm{cv}\right]$, where $\mathrm{cv}$ is chosen large enough to ensure that the event $\tau(X)>$ cv has probability of at most $\alpha$ for all $X \sim P(\theta), \theta \in \Theta_{0}$.

The interest of considering only tests that satisfy (8), rather than the more standard constraint of correct asymptotic null rejection probability for a wide range of primitive assumptions about disturbances that all imply (2), might be motivated in two ways.

On the one hand, one might genuinely worry that the true data generating process happens to be in the set of models that satisfy (2), but the disturbances do not satisfy the primitive conditions. This line of argument then faces the question whether such nonstandard data generating processes are plausible. Especially in a time series context, primitive conditions are often quite opaque (could it be that interest rates are not mixing?), so it is often not clear how and with what arguments one would discuss such a possibility. It is probably fair to say, however, that very general forms of sufficient primitive conditions for central limit theorems and alike were derived precisely because researchers felt uncomfortable assuming more restricted (but still quite general) conditions, so one might say that imposing (8) constitutes only one more step in this progression of generality.

\footnotetext{
${ }^{3}$ Pick $m_{0} \in \mathcal{M}$ with associated probability kernels $\Lambda_{T}^{0}=\left\{\lambda_{T}^{0}(\theta): \theta \in \Theta\right\}$. The family of probability measures $\Lambda_{T}=\left\{\lambda_{T}(\theta): \theta \in \Theta\right\}$ with $\lambda_{T}(\theta)=\lambda_{T}^{0}(\theta)$ for $\theta \neq \theta_{G}$ and $\lambda_{T}\left(\theta_{G}\right)=G_{T}$ then also forms a probability kernel and defines a model $m \in \mathcal{M}$.
} 
Unit RoOT TeST EXAMPLE-CONTINUED: The literature has developed a large number of sufficient conditions on the disturbances $\nu_{T, t}$ that imply $\hat{J}_{T}(\cdot) \rightsquigarrow J_{c}(\cdot)$; see, for instance, McLeish (1974) for a martingale difference sequence framework, Wooldridge and White (1988) for mixing conditions, Phillips and Solo (1992) for linear process assumptions, and Davidson (2002) for near-epoch dependence. Arguably, when invoking such assumptions, researchers do not typically have a specific data generating process in mind that is known to satisfy the conditions; rather there is great uncertainty about the true data generating process, and the hope is that by deriving tests that are valid for a large class of data generating processes, the true model is also covered. The primitive conditions are, therefore, quite possibly not a reflection of what researchers are sure is true about the data generating process, but rather an attempt to assume little so as to gain robustness. In that perspective, it seems quite natural to impose that the asymptotic rejection probability is no larger than the nominal level for all models that satisfy $\hat{J}_{T}(\cdot) \rightsquigarrow W(\cdot)$. In fact, Stock (1994), White (2001, p. 179), Breitung (2002), Davidson (2002, 2009), and Müller (2008) defined the unit root null hypothesis in terms of the convergence $T^{-1 / 2} u_{T, \backslash \cdot T\rfloor} \rightsquigarrow \omega W(\cdot)$, making the requirement (8) quite natural (although $\hat{J}_{T}(\cdot) \rightsquigarrow W(\cdot)$ under the null hypothesis also requires consistency of the long-run variance estimator $\hat{\omega}_{T}^{2}$ as an additional assumption; see Müller (2007) for discussion).

On the other hand, one might argue that the only purpose of an asymptotic analysis is to generate approximations for the small sample under study. In that perspective, it is irrelevant whether interest rates are indeed mixing or not, and the only interesting question becomes whether asymptotic properties derived under an assumption of mixing are useful approximations for the small sample under study. So even in an i.i.d. setting, one might be reluctant to fully exploit all restrictions of a given model - not because it would not be true that with a very large data set, a fully efficient test would be excellent, but because asymptotics might be a poor guide to the behavior of such a test in the sample under study. Focus on the class $\mathfrak{C}$ is then motivated by a concern that additional asymptotic implications of the primitive conditions beyond (2) are potentially poor approximations for the sample under study, and attempts to exploit them may lead to nontrivial size distortions.

LOW-FREQUENCY UNIT RoOT TeST EXAMPLE: Müller and Watson (2008b) argued that in a macroeconomic context, it makes sense to take asymptotic implications of standard models of low frequency variability seriously only over frequencies below the business cycle. So in particular, when $u_{T, t}$ is modelled as local to unity, then the usual asymptotic implication is the functional convergence $T^{-1 / 2} u_{T,\lfloor\cdot T\rfloor} \rightsquigarrow \omega J_{c}(\cdot)$. Müller and Watson (2008b) instead derived a 
scale invariant (we discuss invariance in Section 3.2) point-optimal unit root test that only assumes a subset of this convergence, that is,

$$
\left\{T^{-3 / 2} \sum_{t=1}^{T} \psi_{l}(t / T) u_{T, t}\right\}_{l=1}^{q} \rightsquigarrow\left\{\omega \int_{0}^{1} \psi_{l}(s) J_{\theta}(s) d s\right\}_{l=1}^{q},
$$

where $\psi_{l}(s)=\sqrt{2} \cos (\pi l s)$ and $q$ is some fixed number. No additional (and potentially hard to interpret) assumption is made about the existence of a particular consistent estimator $\hat{\omega}_{T}^{2}$ of $\omega^{2}$. The number $q$ is chosen so that the frequency of the weight functions $\psi_{l}, l=1, \ldots, q$, are below business cycle frequency for the span of the sample under study. The rationale is that picking $q$ larger would implicitly imply a flat spectrum for $\Delta u_{T, t}=u_{T, t}-u_{T, t-1}$ in the I(1) model over business cycle frequencies, which is not an attractive assumption for macroeconomic data. So even if one were certain that for a long enough span of observations, the functional convergence $T^{-1 / 2} u_{T, \downarrow \cdot T\rfloor} \rightsquigarrow \omega J_{c}(\cdot)$ becomes a good approximation eventually, it does not seem well advised to exploit its implications beyond (9) for the sample under study.

\subsection{Main Result}

In addition to the constraint (8), we allow for the possibility that tests $\varphi_{T}$ are restricted to possess further asymptotic properties. In particular, we consider

$$
\begin{aligned}
& \liminf _{T \rightarrow \infty} \int \varphi_{T} d F_{T}(m, \theta) \geq \pi_{0}(\theta) \quad \text { for all } m \in \mathcal{M}, \theta \in \Theta_{1}, \\
& \lim _{T \rightarrow \infty} \int\left(\varphi_{T}-\alpha\right)\left(f_{S} \circ h_{T}\right) d F_{T}(m, \theta)=0 \\
& \quad \text { for all } m \in \mathcal{M}, \theta \in \bar{\Theta}_{0}, \text { and } f_{S} \in \mathcal{F}_{S} .
\end{aligned}
$$

The constraints (10) and (11) are asymptotic analogues of the constraints (5) and (6) in the limiting problem introduced above. So setting $\pi_{0}=\alpha$, for instance, imposes asymptotic unbiasedness of the test $\varphi_{T}$ in the sense that for all models $m \in \mathcal{M}$, the asymptotic rejection probability of $\varphi_{T}$ under the alternative is not smaller than the nominal level. The formulation (11) of "asymptotic conditional similarity" is convenient, as it avoids explicit limits of conditional distributions; see Jansson and Moreira (2006) for discussion and references. Also, without loss of generality, we can always impose (10) and (11), since with $\pi_{0}=0$ and $\mathcal{F}_{S}=\{0\}$, they do not constrain the tests $\varphi_{T}$ in any way.

The main result of this paper is that in the class of tests that satisfy (8), efficient tests in the limiting problem $\varphi_{S}^{*}$, evaluated at sample analogues with $X$ replaced by $X_{T}=h_{T}\left(Y_{T}\right)$, are asymptotically efficient.

THEOREM 1: Let $\varphi_{S}^{*}: S \mapsto[0,1]$ be a level- $\alpha$ test in the limiting problem (3) that maximizes weighted average power (4) subject to (5) and (6). Suppose $\varphi_{S}^{*}$ is 
$\mu_{P}$ almost everywhere continuous and define $\hat{\varphi}_{T}^{*}: \mathbb{R}^{n T} \mapsto[0,1]$ as $\hat{\varphi}_{T}^{*}=\varphi_{S}^{*} \circ h_{T}$. Then the following statements hold:

(i) $\hat{\varphi}_{T}^{*}$ satisfies (8), (10), and (11), and $\lim _{T \rightarrow \infty} \operatorname{WAP}_{T}\left(\hat{\varphi}_{T}^{*}, m\right)=\operatorname{WAP}\left(\varphi_{S}^{*}\right)$ for all $m \in \mathcal{M}$.

(ii) For any test $\varphi_{T}: \mathbb{R}^{n T} \mapsto[0,1]$ satisfying (8), (10), and (11),

$$
\limsup _{T \rightarrow \infty} \operatorname{WAP}_{T}\left(\varphi_{T}, m\right) \leq \operatorname{WAP}\left(\varphi_{S}^{*}\right) \quad \text { for all } m \in \mathcal{M} \text {. }
$$

Unit Root TeST EXAMPLE-CONTINUED: The function $\varphi_{S}^{*}: D_{[0,1]} \mapsto[0,1]$ is continuous at almost all realizations of $W$, so that part (i) of Theorem 1 shows that the test $\hat{\varphi}_{T}^{*}\left(Y_{T}\right)=\varphi_{S}^{*}\left(\hat{J}_{T}(\cdot)\right)=\mathbf{1}\left[\exp \left[-\frac{1}{2} c_{1}\left(\hat{J}_{T}(1)^{2}-1\right)-\right.\right.$ $\left.\left.\frac{1}{2} c_{1}^{2} \int \hat{J}_{T}(s)^{2} d s\right]>\mathrm{cv}\right]$ has asymptotic null rejection probability equal to the nominal level and asymptotic weighted average power equal to $P\left(L\left(J_{c_{1}}\right)>\mathrm{cv}\right)$ for all models in $\mathcal{M}$, that is, models that satisfy $\hat{J}_{T}(\cdot)=T^{-1 / 2} \hat{\omega}_{T}^{-1} u_{T,\lfloor\cdot T\rfloor} \rightsquigarrow W(\cdot)$ and $\hat{J}_{T}(\cdot) \rightsquigarrow J_{c}(\cdot)$. Note that $\hat{\varphi}_{T}^{*}\left(Y_{T}\right)$ is asymptotically equivalent to the efficient unit root test statistic derived by ERS, so the contribution of part (i) of Theorem 1 for the unit root testing example is only to point out that $\hat{\varphi}_{T}^{*}$ has the same asymptotic properties under the null and alternative hypothesis for all models in $\mathcal{M}$.

The more interesting finding is part (ii) of Theorem 1: For any unit root test that has higher asymptotic power than $\hat{\varphi}_{T}^{*}$ for any model $m_{1} \in \mathcal{M}$, that is, some model for which $\hat{J}_{T}(\cdot) \rightsquigarrow J_{c}(\cdot)$, there exists another model $m_{0} \in \mathcal{M}$ for which the test has asymptotic null rejection probability larger than the nominal level. In other words, ERS's test is point-optimal in the class of tests satisfying (8). There is thus a sense in which ERS's test is better than, say, a test based on the Dickey and Fuller (1979) $t$-test type statistic $\left(\hat{J}_{T}(1)^{2}-1\right) /\left(\int \hat{J}_{T}(s)^{2} d s\right)^{1 / 2}$ (which is an element of the class of tests defined by (8)) also without an assumption of Gaussian disturbances.

The proof of part (i) of Theorem 1 follows from the definition of weak convergence, the continuous mapping theorem, and dominated convergence. To gain some intuition for part (ii), consider first the case where the hypotheses are simple, $\Theta_{0}=\left\{\theta_{0}\right\}$ and $\Theta_{1}=\left\{\theta_{1}\right\}$, and (10) and (11) do not bind. Let $L: S \mapsto \mathbb{R}$ be the Radon-Nikodym derivative of $P\left(\theta_{1}\right)$ with respect to $P\left(\theta_{0}\right)$, so that by the Neyman-Pearson lemma, $\varphi_{S}^{*}$ rejects for large values of $L$. For simplicity, assume that $L^{i}=1 / L$ is continuous and bounded. The central idea is to take, for any $m_{1} \in \mathcal{M}$, the implied distribution $F_{T}\left(m_{1}, \theta_{1}\right)$ of $Y_{T}$ and to "tilt" the probabilities according to $L^{i} \circ h_{T}$ to construct a corresponding distribution $G_{T}$ so that $h_{T} G_{T} \rightsquigarrow P\left(\theta_{0}\right)$. This tilted probability distribution needs to integrate to 1 , so let $\kappa_{T}=\int\left(L^{i} \circ h_{T}\right) d F_{T}\left(m, \theta_{1}\right)=\int L^{i} d P_{T}\left(m, \theta_{1}\right)$ and define the measure $G_{T}$ on $\mathfrak{B}\left(\mathbb{R}^{n T}\right)$ via $\int_{A} d G_{T}=\kappa_{T}^{-1} \int_{A}\left(L^{i} \circ h_{T}\right) d F_{T}\left(m, \theta_{1}\right)$ for all $A \in \mathfrak{B}\left(\mathbb{R}^{n T}\right)$. By construction, under $G_{T}$, the function $h_{T}$ induces the measure 
$Q_{T}$ on $S$, where $Q_{T}$ satisfies $\int \vartheta d Q_{T}=\kappa_{T}^{-1} \int \vartheta L^{i} d P_{T}\left(m, \theta_{1}\right)$ for any bounded and continuous function $\vartheta: S \mapsto \mathbb{R}$. Furthermore, the $S \mapsto \mathbb{R}$ functions $\vartheta L^{i}$ and $L^{i}$ are bounded and continuous, so that $P_{T}\left(m, \theta_{1}\right) \rightsquigarrow P\left(\theta_{1}\right)$ implies $\kappa_{T} \rightarrow \int L^{i} d P\left(\theta_{1}\right)=\int L^{i} L d P\left(\theta_{0}\right)=\int d P\left(\theta_{0}\right)=1$ and $\int \vartheta L^{i} d P_{T}\left(m, \theta_{1}\right) \rightarrow$ $\int \vartheta L^{i} d P\left(\theta_{1}\right)=\int \vartheta d P\left(\theta_{0}\right)$, so that $h_{T} G_{T} \rightsquigarrow P\left(\theta_{0}\right)$. Thus, by (8), $\limsup _{T \rightarrow \infty} \int \varphi_{T} d G_{T} \leq \alpha$. Furthermore, by construction, the Radon-Nikodym derivative between $G_{T}$ and $F_{T}\left(m, \theta_{1}\right)$ is given by $\kappa_{T}\left(L \circ h_{T}\right)$. Therefore, by the Neyman-Pearson lemma, the best test of $\tilde{H}_{0}: Y_{T} \sim G_{T}$ against $\tilde{H}_{1}: Y_{T} \sim$ $F_{T}\left(m, \theta_{1}\right)$ rejects for large values of $L \circ h_{T}$, and no test can have a better asymptotic level and power trade-off than this sequence of optimal tests. But $\hat{\varphi}_{T}^{*}$ also rejects for large values of $L \circ h_{T}$ and has the same asymptotic null rejection probability, and the result follows.

In the more general case where $\Theta_{0}$ is composite, and (10) and (11) potentially bind, one can similarly construct, for any distribution $F_{T}\left(m_{1}, \theta_{1}\right), m_{1} \in$ $\mathcal{M}$, a corresponding model $m_{0} \in \mathcal{M}$ via a tilting by $L^{i}(\theta) \circ h_{T}$ for $\theta \in \Theta$, where $L^{i}(\theta)$ is the Radon-Nikodym derivative of $P(\theta)$ with respect to $P\left(\theta_{1}\right)$. By construction, $X_{T}$ is a sufficient statistic for distinguishing $\tilde{H}_{0}: Y_{T} \sim F_{T}\left(m_{0}, \theta\right)$, $\theta \in \Theta_{0}$ against $\tilde{H}_{1}: Y_{T} \sim F_{T}\left(m_{0}, \theta_{1}\right)=F_{T}\left(m_{1}, \theta_{1}\right)$. Standard arguments thus imply that it suffices to consider tests that are functions of $X_{T}$. But the testing problem involving $X_{T}$ is essentially the same as that involving $X$, since the likelihood ratio statistic of $X_{T} \sim P_{T}\left(m_{0}, \theta_{1}\right)$ against $X_{T} \sim P_{T}\left(m_{0}, \theta\right)$ is proportional to $L(\theta)$, with a factor of proportionality that converges to unity as $T \rightarrow \infty$. This again suggests that one cannot do better than the best in the limiting problem under the constraints (5) and (6).

\subsection{Discussion}

Comment 1. Recall how the 14 papers mentioned in the Introduction derive an asymptotically efficient and robust test: Initially, restrict attention to the canonical parametric version of the model of interest, usually with Gaussian i.i.d. disturbances. Call this model $m^{*}$, so that $Y_{T} \sim F_{T}\left(m^{*}, \theta\right)$ and, for simplicity, consider the problem of testing the simple hypotheses $H_{0}: \theta=\theta_{0}$ against $H_{1}: \theta=\theta_{1}$. In this parametric model, $F_{T}\left(m^{*}, \theta_{1}\right)$ is absolutely continuous with respect to $F_{T}\left(m^{*}, \theta_{0}\right)$ and the small sample likelihood ratio statistic $\mathrm{LR}_{T}$ can be derived. The small sample optimal test in model $m^{*}$ thus rejects for large values of $\mathrm{LR}_{T}$. Express $\mathrm{LR}_{T}$ (up to asymptotically negligible terms) as a continuous function $L: S \mapsto \mathbb{R}$ of a random element $X_{T}^{*}=h_{T}^{*}\left(Y_{T}\right)$ that converges weakly under the null and contiguous alternative: $\operatorname{LR}_{T}=L\left(X_{T}^{*}\right)+o_{p}(1)$, where $X_{T}^{*} \rightsquigarrow X$ with $X \sim P(\theta)$. Thus, by the continuous mapping theorem, the likelihood ratio statistic also converges weakly under the null and alternative, $\mathrm{LR}_{T} \rightsquigarrow \mathrm{LR} \sim L P(\theta)$, and the asymptotic critical value is computed from the distribution $L P\left(\theta_{0}\right)$. Furthermore, an asymptotically robust test statistic is given by $L\left(X_{T}\right)$, where $X_{T}=h_{T}\left(Y_{T}\right)$ is a "robustified" version of $X_{T}^{*}$ such that 
$X_{T} \rightsquigarrow X \sim P(\theta)$ in many models $m$ of interest, including $m^{*}$ (whereas typically $X_{T}^{*}=h_{T}^{*}\left(Y_{T}\right) \ngtr \leftrightarrow X$ for some plausible models).

UNIT RoOT TEST EXAMPLE-CONTINUED: With i.i.d. standard normal driving disturbances, the small sample efficient unit root test rejects for large values of $\mathrm{LR}_{T}=\exp \left[-\frac{1}{2} c_{1} T^{-1}\left(u_{T, T}^{2}-\sum_{t=1}^{T}\left(\Delta u_{T, t}\right)^{2}\right)-\frac{1}{2} c_{1}^{2} T^{-2} \sum_{t=1}^{T} u_{T, t-1}^{2}\right]$; compare Dufour and King (1991). With $h_{T}^{*}\left(Y_{T}\right)=X_{T}^{*}=T^{-1 / 2} u_{T,\lfloor\cdot T\rfloor} \rightsquigarrow J_{c}(\cdot)$, we thus have $\mathrm{LR}_{T}=L\left(X_{T}^{*}\right)+o_{p}(1)$ with $L(x)=\exp \left[-\frac{1}{2} c_{1}\left(x(1)^{2}-1\right)-\right.$ $\left.\frac{1}{2} c_{1}^{2} \int_{0}^{1} x(s)^{2} d s\right]$. The asymptotically robustified test that allows for serially correlated and non-Gaussian disturbances is based on $L\left(X_{T}\right)$, where $X_{T}=\hat{J}_{T}(\cdot)=$ $T^{-1 / 2} \hat{\omega}_{T}^{-1} u_{T, \backslash \cdot T\rfloor}$.

The end product of this standard approach is a test based on the statistic $L\left(X_{T}\right)$, with critical value computed from the distribution $L P\left(\theta_{0}\right)$. Now, generically, this test is identical to the test $\hat{\varphi}_{T}^{*}$ of Theorem 1 . This follows from a general version of Le Cam's third lemma (see, for instance, Lemma 27 of Pollard (2001)): If the measures $F_{T}\left(m^{*}, \theta_{0}\right)$ and $F_{T}\left(m^{*}, \theta_{1}\right)$ are contiguous with likelihood ratio statistic $\mathrm{LR}_{T}$, and under $Y_{T} \sim F_{T}\left(m^{*}, \theta_{0}\right),\left(\mathrm{LR}_{T}, X_{T}^{*}\right) \rightsquigarrow(L(X), X)$ with $X \sim P\left(\theta_{0}\right)$ and some function $L: S \mapsto \mathbb{R}$, then under $Y_{T} \sim F_{T}\left(m^{*}, \theta_{1}\right)$, $X_{T}^{*} \rightsquigarrow X \sim Q$ and the Radon-Nikodym derivative of $Q$ with respect to $P\left(\theta_{0}\right)$ is equal to $L$. So if it is known that under $Y_{T} \sim F_{T}\left(m^{*}, \theta_{1}\right), X_{T}^{*} \rightsquigarrow X \sim P\left(\theta_{1}\right)$, then it must be that $Q=P\left(\theta_{1}\right)$, and $L(X)$ is recognized as the NeymanPearson test statistic of the limiting problem $H_{0}^{l p}: \theta=\theta_{0}$ against $H_{1}^{l p}: \theta=\theta_{1}$ with $X \sim P(\theta)$ observed. The test that rejects for large values of $L\left(X_{T}\right)$ is thus simply the efficient test of this limiting problem, evaluated at sample analogues. This explains why in the unit root example the test $\hat{\varphi}_{T}^{*}$ of Theorem 1 had to be asymptotically equivalent to ERS's statistic.

Comment 2. This standard construction of tests starting from the canonical parametric model $m^{*}$, that is, rejecting for large values of $L\left(X_{T}\right)$, is by construction asymptotically efficient in model $m^{*}$ and, by part (i) of Theorem 1 , it has the same asymptotic rejection probabilities for all models $m \in \mathcal{M}$. This does not, however, make the test $L\left(X_{T}\right)$ necessarily overall asymptotically efficient: It might be that there exists another test with the same asymptotic power in model $m^{*}$ and higher asymptotic power for at least some other model $m_{1} \in \mathcal{M}$. The semiparametric efficient unit root test by Jansson (2008) is an example of a test with the same asymptotic power as ERS's test for Gaussian i.i.d. disturbances and higher asymptotic power for some other driving disturbances.

Now part (ii) of Theorem 1 shows that whenever a test has higher asymptotic power than $\hat{\varphi}_{T}^{*}$ for some model $m_{1} \in \mathcal{M}$, then it cannot be a member of $\mathfrak{C}$. Any partial adaption to models $m_{1} \neq m^{*}$, if successful, necessarily implies the existence of a model $m_{0} \in \mathcal{M}$ for which the test has asymptotic rejection probability larger than the nominal level. In particular, Theorem 1 implies the existence 
of a double array process $\left(u_{T, 1}, \ldots, u_{T, T}\right)^{\prime}$ satisfying $T^{-1 / 2} \hat{\omega}_{T}^{-1} u_{T,\lfloor\cdot T\rfloor} \rightsquigarrow W(\cdot)$ for which Jansson's (2008) test has asymptotic rejection probability greater than the nominal level.

In other words, Theorem 1 shows $\hat{\varphi}_{T}^{*}$ to be an asymptotically efficient test in the class $\mathfrak{C}$, because no test can exist with higher asymptotic (weighted average) power for any model $m \in \mathcal{M}$.

Comment 3. In this sense, Theorem 1 implies a particular version of an asymptotic essentially complete class result for the hypothesis test (1): Set $\pi_{0}$ in (10) equal to the power function of an admissible test in the limiting problem (3), so that (10) effectively determines $\varphi_{S}^{*}$. The theorem then shows that no test $\varphi_{T}$ in $\mathfrak{C}$ can have higher asymptotic power than $\hat{\varphi}_{T}^{*}$ uniformly over $\theta \in \Theta_{1}$. As long as all admissible $\varphi_{S}^{*}$ are $\mu_{P}$ almost everywhere continuous, the resulting tests $\hat{\varphi}_{T}^{*}$ thus form an essentially complete subset of asymptotically admissible tests of $\mathfrak{C}$.

In particular, if a uniformly most powerful test exists in the limiting problem, then $\varphi_{S}^{*}$ is this test for any weighting function $w$, and repeated application of Theorem 1 with $w$ having point mass for any $\theta \in \Theta_{1}$ then shows that the test $\hat{\varphi}_{T}^{*}$ is correspondingly asymptotically uniformly most powerful.

Comment 4. As discussed in the Introduction, an important subset of tests that are automatically members of $\mathfrak{C}$ are those asymptotic level- $\alpha$ tests that can be written as a (sufficiently) continuous function of $X_{T}$ : Let $\varphi_{c}: S \mapsto[0,1]$ be a $\mu_{P}$ almost everywhere continuous function. Then $\varphi_{T}=\varphi_{c} \circ h_{T}$ has asymptotic rejection probability equal to $\int \varphi_{c} d P(\theta) \leq \alpha$ for all $\theta \in \Theta_{0}$ by the continuous mapping theorem and thus satisfies (8) whenever it is of level $\alpha$ in the canonical model. As a corollary to Theorem $1, \hat{\varphi}_{T}^{*}$ is thus asymptotic weighted average power maximizing among all such tests, and this also holds if $\varphi_{c}\left(\right.$ and $\varphi_{S}^{*}$ in $\left.\hat{\varphi}_{T}^{*}=\varphi_{S}^{*} \circ h_{T}\right)$ is restricted by (5) and (6). This reasoning can be generalized further by considering the set of tests $\varphi_{T}: \mathbb{R}^{n T} \mapsto[0,1]$ that are amenable to a continuous mapping theorem for a sequence of functions (cf. Theorem 1.11.1 in van der Vaart and Wellner (1996)) so that their limiting behavior is still governed by some $\mu_{P}$ almost everywhere continuous function $\varphi_{c}$.

Reliance on $\hat{\varphi}_{T}^{*}$ can therefore be motivated also if (8) is not an appealing restriction. In nonlinear and/or dynamic models, it might be easier to think about high level weak convergences, rather than small sample distributions, even under strong parametric assumptions. The problem of testing for parameter instability in a general GMM framework, as considered by Sowell (1996) and discussed in detail in Section 4.3 below, or the weak instrument problem in a general GMM framework, as considered by Stock and Wright (2000), arguably fall into this class. In these problems, one natural starting point is tests that are (sufficiently) continuous functions of the weakly converging statistics, and Theorem 1 identifies the best such test. 
UNIT RoOT TeST EXAMPLE-CONTINUED: Stock (1999) exactly considered tests of the form $\varphi_{c}\left(\hat{J}_{T}(\cdot)\right)$ in the notation developed here. Theorem 1 thus also identifies $\varphi_{S}^{*}\left(\hat{J}_{T}(\cdot)\right)$ as the point-optimal test in his class.

Comment 5. When $\Theta$ is of finite dimension, one might call the set of models $\mathcal{M}$ "semiparametric," with the weak convergence assumption (2) constituting the nonparametric aspect. This raises the question of how Theorem 1 relates to the more traditional concept of semiparametric efficient inference. In the following discussion, we draw this comparison for a simple model with locally asymptotically normal (LAN) likelihood ratios.

For expositional ease, consider $T$ scalar observations $Y_{T}=\left(y_{T, 1}, \ldots, y_{T, T}\right)^{\prime}$ with mean $E\left[y_{T, t}\right]=\mu$ and variance $E\left[\left(y_{T, t}-\mu\right)^{2}\right]=1$, where the hypotheses are $H_{0}: \mu=0$ against $H_{1}: \mu>0$. Reparametrize $\theta=\sqrt{T} \mu$, so that in terms of $\theta$, the problem becomes $H_{0}: \theta=0$ against $H_{1}: \theta>0$. It is natural to take the sample mean $\bar{y}_{T}=T^{-1} \sum_{t=1}^{T} y_{T, t}$ as the basis for inference, so consider the monotone transformation $X_{T}=h_{T}\left(Y_{T}\right)=\sqrt{T} \bar{y}_{T} \rightsquigarrow X \sim \mathcal{N}(\theta, 1)$, where the convergence stems from a central limit theorem. The likelihood ratio statistic of $X \sim \mathcal{N}\left(\theta_{1}, 1\right)$ against $X \sim \mathcal{N}(0,1)$ equals $L(X)=\exp \left[\theta_{1} X-\frac{1}{2} \theta_{1}^{2}\right]$, so that by the Neyman-Pearson lemma, $\varphi_{S}^{*}(X)=\mathbf{1}[X>1.645]$ is the the uniformly most powerful $5 \%$ level test in the limiting problem of observing $X \sim \mathcal{N}(\theta, 1)$. Thus, by Theorem $1, \hat{\varphi}_{T}^{*}\left(Y_{T}\right)=\varphi_{S}^{*}\left(X_{T}\right)$ is the best $5 \%$ level test among all tests whose asymptotic null rejection probability is no greater than $5 \%$ whenever $X_{T} \rightsquigarrow \mathcal{N}(0,1)$.

Ignoring the unboundedness of $L^{i}=1 / L$ for simplicity, the basic argument in the proof of Theorem 1 starts from any alternative model $F_{T}\left(m, \theta_{1}\right)$ (so that under $\left.F_{T}\left(m, \theta_{1}\right), X_{T} \sim \mathcal{N}\left(\theta_{1}, 1\right)\right)$ and constructs a corresponding null model $G_{T}$ by tilting the probabilities according to $L^{-1} \circ h_{T}=\exp \left[-\theta_{1} X_{T}+\frac{1}{2} \theta_{1}^{2}\right]$, that is, $\int_{A} d G_{T}=\kappa_{T}^{-1} \int_{A}\left(L^{i} \circ h_{T}\right) d F_{T}\left(m, \theta_{1}\right)$ for all $A \in \mathfrak{B}\left(\mathbb{R}^{T}\right)$, where $\kappa_{T} \rightarrow 1$ insures that $\int_{\mathbb{R}^{T}} d G_{T}=1$. If under the alternative model $F_{T}\left(m, \theta_{1}\right),\left\{y_{T, t}\right\}_{t=1}^{T}$ is i.i.d. with $\log$ density $y \mapsto g\left(y-T^{-1 / 2} \theta_{1}\right)$, we obtain

$$
\begin{aligned}
\int_{A} d G_{T}= & \int_{A} \prod_{t=1}^{T} \exp \left[g\left(y_{T, t}-T^{-1 / 2} \theta_{1}\right)-T^{-1 / 2} \theta_{1} y_{T, t}\right. \\
& \left.+\frac{1}{2} T^{-1} \theta_{1}^{2}-T^{-1} \ln \kappa_{T}\right] d Y_{T}
\end{aligned}
$$

so that the tilted model $G_{T}$ is also i.i.d., with $\log$ density $g\left(y-T^{-1 / 2} \theta_{1}\right)-$ $T^{-1 / 2} \theta_{1} y+\frac{1}{2} T^{-1} \theta_{1}^{2}-T^{-1} \ln \kappa_{T}$.

Now consider the analysis of inference about the mean in an i.i.d. scalar series using traditional semiparametric techniques. Write $\varepsilon$ for a mean zero random variable with distribution $y_{T, t}-\mu$, and embed the log density $g$ of $\varepsilon$ in a smooth parametric model $g_{\eta}$ with parameter $\eta \in \mathbb{R}$. Under the local 
parametrization $(\theta, v)=\sqrt{T}\left(\mu, \eta-\eta_{0}\right)$ and some regularity conditions, we obtain that the log-likelihood process satisfies

$$
\begin{gathered}
\sum_{t=1}^{T}\left(g_{\eta_{0}+T^{-1 / 2}}\left(y_{T, t}-T^{-1 / 2} \theta\right)-g_{\eta_{0}}\left(y_{T, t}\right)\right) \\
=S_{T}^{\prime}\left(\begin{array}{l}
\theta \\
v
\end{array}\right)-\frac{1}{2}\left(\begin{array}{c}
\theta \\
v
\end{array}\right)^{\prime} \mathcal{I}\left(\begin{array}{l}
\theta \\
v
\end{array}\right)+o_{p}(1),
\end{gathered}
$$

where $S_{T}=T^{-1 / 2}\left(\sum_{t=1}^{T} \ell_{\mu}\left(y_{T, t}\right), \sum_{t=1}^{T} \ell_{\eta}\left(y_{T, t}\right)\right)^{\prime} \rightsquigarrow \mathcal{N}(0, \mathcal{I}), \mathcal{I}=\left(\left(\mathcal{I}_{\mu \mu}, \mathcal{I}_{\mu \eta}\right)^{\prime}\right.$, $\left.\left(\mathcal{I}_{\mu \eta}, \mathcal{I}_{\eta \eta}\right)^{\prime}\right)$, and $\ell_{\mu}$ and $\ell_{\eta}$ are $\mathbb{R} \mapsto \mathbb{R}$ functions with $E\left[\ell_{\mu}(\varepsilon)\right]=E\left[\ell_{\eta}(\varepsilon)\right]=$ $0, E\left[\ell_{\mu}(\varepsilon)^{2}\right]=\mathcal{I}_{\mu \mu}, E\left[\ell_{\eta}(\varepsilon)^{2}\right]=\mathcal{I}_{\eta \eta}, E\left[\ell_{\mu}(\varepsilon) \ell_{\eta}(\varepsilon)\right]=\mathcal{I}_{\mu \eta}$, and $E\left[\varepsilon\left(\ell_{\mu}(\varepsilon)\right.\right.$, $\left.\left.\ell_{\eta}(\varepsilon)\right)\right]=(1,0)$. It is useful to think of $\ell_{\mu}$ and $\ell_{\eta}$ as appropriate generalizations of the usual score functions $d g_{\eta_{0}}(y-\mu) /\left.d \mu\right|_{\mu=0}$ and $d g_{\eta}(y) /\left.d \eta\right|_{\eta=\eta_{0}}$, respectively, so that under weak regularity conditions, $E\left[\varepsilon\left(\ell_{\mu}(\varepsilon), \ell_{\eta}(\varepsilon)\right)\right]=(1,0)$ follows from the identity $\int y \exp g_{\eta}(y-\mu) d y=\mu$. Furthermore, by Le Cam's third lemma, $S_{T} \rightsquigarrow \mathcal{N}\left(\mathcal{I}(\theta, v)^{\prime}, \mathcal{I}\right)$ when the true log density of $y_{T, t}$ is given by $g_{\eta_{0}+T^{-1 / 2} v}\left(y-T^{-1 / 2} \theta\right)$. For any $\theta_{1}>0$, consider the best test of the simple null hypothesis $H_{0}:(\theta, v)=\left(0, v_{0}\right)$ with $v_{0}=\mathcal{I}_{\eta \eta}^{-1} \mathcal{I}_{\mu \eta} \theta_{1}$ against the single alternative $H_{1}:(\theta, v)=\left(\theta_{1}, 0\right)$. In large samples, the Neyman-Pearson test rejects for large values of $\left(\left(\theta_{1},-v_{0}\right) \mathcal{I}\left(\theta_{1},-v_{0}\right)^{\prime}\right)^{-1 / 2}\left(\theta_{1},-v_{0}\right) S_{T} \rightsquigarrow \mathcal{N}(a, 1)$, where $a=0$ under the null hypothesis and $a=\theta_{1} \sqrt{\mathcal{I}_{\mu \mu}-\mathcal{I}_{\mu \eta}^{2} / \mathcal{I}_{\eta \eta}}$ under the alternative. Because $a=0$ for any local parameter of the form $(0, v)$, the choice of $v_{0}=\mathcal{I}_{\eta \eta}^{-1} \mathcal{I}_{\mu \eta} \theta_{1}$ is least favorable, so that by Theorem 7 of Lehmann (1986, p. 104), this test is the best test against $H_{1}$ also under the composite null hypothesis that leaves $v$ unrestricted. Furthermore, this test does not depend on $\theta_{1}$ (since it is is equivalent to rejecting for large values of $\left.\left(\mathcal{I}_{\eta \eta},-\mathcal{I}_{\mu \eta}\right) S_{T}\right)$ and is thus uniformly most powerful against alternatives of the form $(\theta, 0)$ with $\theta>0$.

Now Stein's (1956) key insight underlying semiparametric efficiency calculations is that inference with $g$ unknown is at least as hard as inference in any of the smooth parametric submodels with parameter $\eta$. Because the power of the best test in any particular submodel increases in $\mathcal{I}_{\mu \mu}-\mathcal{I}_{\mu \eta}^{2} / \mathcal{I}_{\eta \eta}$, the least favorable submodel is the one that minimizes this quantity, subject to the constraint that $E\left[\varepsilon\left(\ell_{\mu}(\varepsilon), \ell_{\eta}(\varepsilon)\right)\right]=(1,0)$. This is solved by $\ell_{\eta}(\varepsilon)=\ell_{\mu}(\varepsilon)-\varepsilon$ for which $\mathcal{I}_{\eta \eta}=\mathcal{I}_{\mu \eta}=\mathcal{I}_{\mu \mu}-1$ and $a=\theta_{1}$ under the alternative. But $X_{T}=\sqrt{T} \bar{y}_{T}$ achieves this noncentrality parameter, so that $\hat{\varphi}_{T}^{*}\left(Y_{T}\right)=\mathbf{1}\left[X_{T}>1.645\right]$ is also the semiparametric efficient $5 \%$ level test.

As just discussed, the rationale for this semiparametric efficiency claim is that for any alternative model with log density $g\left(y-T^{-1 / 2} \theta_{1}\right)$, there exists (or, more precisely, one can arbitrarily well approximate) a least favorable smooth parametric submodel $g_{\eta}$ with $g=g_{\eta_{0}}$ and $\ell_{\eta}(\varepsilon)=\ell_{\mu}(\varepsilon)-\varepsilon$, and a least favorable null value $v_{0}=\mathcal{I}_{\eta \eta}^{-1} \mathcal{I}_{\mu \eta} \theta_{1}=\theta_{1}$ of the parameter $\eta=\eta_{0}+T^{-1 / 2} v_{0}$ so that by (13), the likelihood ratio process satisfies $\sum_{t=1}^{T}\left(g_{\eta_{0}}\left(y_{T, t}-T^{-1 / 2} \theta_{1}\right)-\right.$ 
$\left.g_{\eta_{0}+T^{-1 / 2} \theta_{1}}\left(y_{T, t}\right)\right)=\theta_{1} T^{-1 / 2} \sum_{t=1}^{T} y_{T, t}-\frac{1}{2} \theta_{1}^{2}+o_{p}(1)$. Solving this for the least favorable null log density, we obtain

$$
\begin{aligned}
& \sum_{t=1}^{T} g_{\eta_{0}+T^{-1 / 2} \theta_{1}}\left(y_{T, t}\right) \\
& \quad=\sum_{t=1}^{T}\left(g_{\eta_{0}}\left(y_{T, t}-T^{-1 / 2} \theta_{1}\right)-\theta_{1} T^{-1 / 2} y_{T, t}+\frac{1}{2} T^{-1} \theta_{1}^{2}\right)+o_{p}(1),
\end{aligned}
$$

which is essentially the same as the tilted model (12).

So not only do both approaches to the question of efficient tests lead to the same test $\hat{\varphi}_{T}^{*}\left(Y_{T}\right)=\mathbf{1}\left[X_{T}>1.645\right]$, but also the least favorable null model that "defeats" attempts to learn from the data about $\mu$ through statistics other than $X_{T}$ is (at least approximately) the same in both approaches. The semiparametric efficiency result is arguably sharper, though, as it accommodates the moment condition and i.i.d. property of the observations, whereas Theorem 1 yields a less specific efficiency claim.

Comment 6. One advantage of Theorem 1 relative to the semiparametric efficiency approach is the general nature of its claim. The result requires only minimal regularity conditions beyond the basic weak convergence assumption and covers a wide range of nonstandard testing problems. In contrast, relatively little is known about traditional semiparametric efficiency in models outside the LAN class, and deriving such results is nontrivial and involves additional regularity assumptions (cf. Jansson (2008)).

When Theorem 1 yields a less powerful test than what is obtained from a traditional semiparametric efficiency calculation, one might try to reconcile the results either by assuming additional weak convergences (which potentially increases the power of $\varphi_{S}^{*}$ and $\hat{\varphi}_{T}^{*}$ ) or by considering less constrained semiparametric models (which potentially decreases the power of the semiparametric efficient test). For the latter, inspection of the form of the tilted model $G_{T}$ in the proof of Theorem 1 may suggest an appropriate relaxation of the constraints.

Unit Root TeSt ExAMPLE-CONTINUED: Proceeding as in Rothenberg and Stock (1997) and Jansson (2008) yields that the log of the likelihood ratio statistic for testing $H_{0}: c=0$ against $H_{1}: c=c_{1}$ in an $\operatorname{AR}(1)$ model with coefficient $1-c / T$ and i.i.d. driving errors with the same distribution as $\varepsilon$ satisfies $-c_{1} T^{-1} \sum_{t=1}^{T} \ell_{\mu}\left(\Delta u_{T, t}\right) u_{T, t-1}-\frac{1}{2} c_{1}^{2} \mathcal{I}_{\mu \mu} T^{-2} \sum_{t=1}^{T} u_{T, t-1}^{2}+o_{p}(1)$ with $\varepsilon, \ell_{\mu}$, and $\mathcal{I}_{\mu \mu}$ as defined in Comment 5 above. Heuristically, the tilting by $1 / L$ is approximately equal to a tilting by $1 / \mathrm{LR}_{T}$, and a model tilted by $1 / \mathrm{LR}_{T}$ has joint log-density $\sum_{t=1}^{T}\left[g\left(\Delta u_{T, t}\right)-c_{1} T^{-1}\left(\ell_{\mu}\left(\Delta u_{T, t}\right)-\right.\right.$ $\left.\left.\Delta u_{T, t}\right) u_{T, t-1}-\frac{1}{2} c_{1}^{2} T^{-2}\left(\mathcal{I}_{\mu \mu}-1\right) u_{T, t-1}^{2}\right]+o_{p}(1)$. This is recognized as an expansion of $\sum_{t=1}^{T} g_{\eta_{0}-c_{1} / T}^{M}\left(\Delta u_{T, t}, u_{T, t-1}\right)$ with $\exp \left(g_{\eta}^{M}(y, x)\right)=e^{g(y)}(1+$ 
$\left.\left(\eta-\eta_{0}\right)\left(\ell_{\mu}(y)-y\right) x\right)$ for $\eta \in \mathbb{R}$. Note that $\int \exp \left(g_{\eta}^{M}(y, x)\right) d y=1$ and $\int y \exp \left(g_{\eta}^{M}(y, x)\right) d y=0$ for all $x$ and $\eta$, suggesting that $\sum_{t=1}^{T} g_{\eta_{0}-c_{1} / T}^{M}\left(\Delta u_{T, t}\right.$, $u_{T, t-1}$ ) are the log Markov kernels of a time homogeneous first order Markov martingale for $\left\{u_{T, t}\right\}_{t=1}^{T}$. As $\left(\ell_{\mu}(y)-y\right) x$ is unbounded, though, $g_{\eta}^{M}(y, x)$ is not well defined even in the $T^{-1}$ neighborhood of $\eta_{0}$. But one could presumably employ an appropriate approximation scheme, so that an analysis analogous to Jansson (2008) would yield $\hat{\varphi}_{T}^{*}$ to be the semiparametrically point-optimal unit root tests in the first order time homogeneous Markov model against AR(1) alternatives. A detailed analysis of this conjecture is beyond the scope of this paper.

Comment 7. The weak convergence assumption (2) can be viewed as a way to express regularity one is willing to impose on some inference problem. Implicitly, this is standard practice: invoking standard normal asymptotics for the ordinary least squares (OLS) estimator of the largest autoregressive root $\rho$ is formally justified for any value of $|\rho|<1$, but effectively amounts to the assumption that the true parameter in the sample under study is not close to the local-to-unity region. Similarly, a choice of weak versus strong instrument asymptotics or local versus nonlocal time varying parameter asymptotics expresses knowledge of regularity in terms of weak convergences.

In some instances, it might be natural to express all regularity that one is willing to impose in this form, and Theorem 1 then shows that $\hat{\varphi}_{T}^{*}$ efficiently exploits this information. The i.i.d. nature of (standard) cross sectional data is not easily embedded in a weak convergence statement, so that such a starting point is much more convincing in a time series context. Also, interesting high level weak convergence assumptions are certainly not arbitrary, but derive their plausibility from the knowledge that there exists a range of underlying primitive conditions that would imply them.

If one expresses regularity of a problem in terms of weak convergences, one faces a choice of what to assume. Of course, additional weak convergence assumptions cannot reduce the information about $\theta$ in the limiting problem. But not all weak convergences are relevant for deciding between $H_{0}$ and $H_{1}$ : Whenever the conditional distribution of the additional limiting element does not depend on $\theta$, the efficient test remains unaltered. This holds, for example, for any additional convergence in probability to a constant limiting element whose value does not depend on $\theta$. In the context of unit root tests, for instance, ERS's test remains asymptotically efficient in the sense of Theorem 1 if in addition to $\hat{J}_{T}(\cdot) \rightsquigarrow J_{c}(\cdot)$, the average sample skewness of $\Delta u_{T, t}=u_{T, t}-u_{T, t-1}, T^{-1} \sum_{t=1}^{T}\left(\Delta u_{T, t}\right)^{3}$, is assumed to converge to zero in probability, or that $T^{-1 / 2} \sum_{t=1}^{\lfloor\cdot T\rfloor}\left(\Delta u_{T, t}\right)^{3} \rightsquigarrow W_{\Delta}(\cdot)$ for any $c \geq 0$, where $W_{\Delta}(\cdot)$ is a Wiener process independent of $J_{c}$.

At the same time, one might also be reluctant to impose the full extent of the "usual" weak convergence assumption; in general, this leads to less powerful 
inference. The efficiency claim of Theorem 1 then shows that it is impossible to use data-dependent methods to improve inference for more regular data while still remaining robust in the sense of (8).

Low-FreQuency Unit Root Test EXAMPLE-Continued: Since (9) is strictly weaker than the standard assumption $T^{-1 / 2} u_{T,\lfloor\cdot T\rfloor} \rightsquigarrow \omega J_{c}(\cdot)$, Müller and Watson's (2008b) low-frequency unit root test is less powerful than ERS's test. It is nevertheless point-optimal in the sense of efficiently extracting all regularity contained in the weaker statement (9): Theorem 1 implies that it is impossible to let the data decide whether (9) holds for $q$ larger than assumed (that is, whether the local-to-unity model provides good approximations also over business cycle frequencies), and to conduct more powerful inference if it is, without inducing size distortions for some model satisfying (9).

\section{EXTENSIONS}

\subsection{Consistently Estimable Nuisance Parameters}

Suppose the testing problem (1) involves an additional nuisance parameter $\gamma \in \Gamma$, where $\Gamma$ is a metric space, so that now $Y_{T} \sim F_{T}(m, \theta, \gamma)$, and the null and alternative hypotheses become

$$
H_{0}:(\theta, \gamma) \in\left(\Theta_{0}, \Gamma\right) \text { against } H_{1}:(\theta, \gamma) \in\left(\Theta_{1}, \Gamma\right) .
$$

Suppose $\gamma$ can be consistently estimated by the estimator $\hat{\gamma}_{T}$ under the null and alternative hypothesis. For a fixed value of $\gamma=\gamma_{0}$, that is, when $\Gamma$ is a singleton, this is a special case of what is covered by Theorem 1, as discussed in Comment 7. But when $\Gamma$ is not a singleton, the analysis above is not immediately applicable, because the limiting measures of $X$ were assumed to be mutually absolutely continuous for all parameter values.

Thus denote by $P_{T}^{e}(m, \theta, \gamma)$ (e denotes extended) the distribution of $\left(\hat{\gamma}_{T}, X_{T}\right)=h_{T}^{e}\left(Y_{T}\right)$ when $Y_{T} \sim F_{T}(m, \theta, \gamma)$, so that the weak convergence assumption analogous to (2) now becomes

$$
P_{T}^{e}(m, \theta, \gamma) \rightsquigarrow P^{e}(\theta, \gamma) \quad \text { pointwise for all }(\theta, \gamma) \in(\Theta \times \Gamma),
$$

where $P^{e}(\theta, \gamma)$ is the product measure between the measure $P(\theta, \gamma)$ of $X$ on $\mathfrak{B}(S)$ (which might depend on $\gamma$ and where for all $\gamma \in \Gamma$, the measures $P(\theta, \gamma)$, $\theta \in \Theta$ are equivalent to some measure $\left.\mu_{P}(\gamma)\right)$ and the degenerate probability measure on $\mathfrak{B}(\Gamma)$ that puts all mass on the point $\gamma$. The limiting problem now becomes testing (14) with $X \sim P(\theta, \gamma)$ observed and $\gamma$ known, and optimal tests $\varphi_{S}^{e *}$ in the limiting problem are indexed by $\gamma \in \Gamma, \varphi_{S}^{e *}: \Gamma \times S \mapsto[0,1]$, so that for each $\gamma_{0} \in \Gamma, \varphi_{S}^{e *}\left(\gamma_{0}, \cdot\right)$ is the weighted average power maximizing test of (14) for $\gamma=\gamma_{0}$, possibly subject to constraints of the form (5) and (6).

Now as long as the test $\varphi_{S}^{e *}$ is $\mu_{P}(\gamma)$ almost everywhere continuous for all $\gamma \in \Gamma$, the same arguments as employed in the proof of Theorem 1(i) imply 
that under (15) and for all $\gamma \in \Gamma$, the test $\hat{\varphi}_{T}^{e *}=\varphi_{S}^{e *}\left(\hat{\gamma}_{T}, X_{T}\right)$ has the same asymptotic size and power as $\varphi_{S}^{e *}$ does. Furthermore, one can invoke Theorem 1(ii) to conclude that $\hat{\varphi}_{T}^{e *}$ is efficient when restricting attention to models with $\gamma=\gamma_{0}$ known. Since $\hat{\varphi}_{T}^{e *}$ does not require knowledge of $\gamma$, this shows that $\hat{\varphi}_{T}^{e *}$ is overall efficient. Problems that involve consistently estimable parameters $\gamma$ with an impact on the efficient limiting tests are thus covered by the results of Theorem 1 under an additional assumption of the family of efficient limiting tests, indexed by $\gamma$, to depend on $\gamma$ sufficiently smoothly.

Unit Root TeSt ExAmple-Continued: Instead of $T^{-1 / 2} \hat{\omega}_{T}^{-1} u_{T,\lfloor\cdot T\rfloor}=$ $\hat{J}_{T}(\cdot) \rightsquigarrow J_{c}(\cdot)$, consider the weak convergences $h_{T}^{e}\left(Y_{T}\right)=\left(\hat{\omega}_{T}^{2}, T^{-1 / 2} u_{T,\lfloor\cdot T\rfloor}\right) \rightsquigarrow$ $\left(\omega^{2}, \omega J_{c}(\cdot)\right)$ as a starting point. In the limiting problem, $X=\omega J_{c}(\cdot)$ is observed with $\omega^{2}$ known, and the point-optimal test is of the form $\varphi_{S}^{e *}\left(\omega^{2}, X\right)=$ $\mathbf{1}\left[\exp \left[-\frac{1}{2} c_{1}\left(\omega^{-2} X(1)^{2}-1\right)-\frac{1}{2} c_{1}^{2} \omega^{-2} \int_{0}^{1} X(s)^{2} d s\right]>\mathrm{cv}\right]$. A calculation shows this to be a continuous function $(0, \infty) \times D_{[0,1]} \mapsto \mathbb{R}$ for almost all realizations of $\left(\omega^{2}, J_{0}\right)$, so the test $\varphi_{S}^{e *}\left(\hat{\omega}_{T}^{2}, T^{-1 / 2} u_{T,\lfloor\cdot T\rfloor}\right)$ is asymptotically efficient among all unit root tests with correct asymptotic null rejection probability whenever $\left(\hat{\omega}_{T}^{2}, T^{-1 / 2} u_{T,\lfloor\cdot T\rfloor}\right) \rightsquigarrow\left(\omega^{2}, \omega J_{0}(\cdot)\right)$ against all models satisfying $\left(\hat{\omega}_{T}^{2}, T^{-1 / 2} u_{T,\lfloor\cdot T\rfloor}\right) \rightsquigarrow\left(\omega^{2}, \omega J_{c_{1}}(\cdot)\right)$.

\subsection{Invariance}

The majority of efficient tests for nonstandard problems cited in the Introduction rely on invariance considerations. In the framework here, invariance may be invoked at two levels: On one hand, one might consider a weak convergence as a starting point that is a function of a small sample maximal invariant; on the other hand, invariance might instead be employed in the limiting problem as a way to deal with nuisance parameters. This subsection discusses the link between these two notions, and the interaction of the concept of invariance with the efficiency statements of Theorem 1.

The first case is entirely straightforward: suppose $\phi_{T}\left(Y_{T}\right)$ with $\phi_{T}: \mathbb{R}^{n T} \mapsto$ $\mathbb{R}^{n T}$ is a maximal invariant to some group of transformations. By Theorem 1 of Lehmann (1986, p. 285), all invariant tests can be written as functions of a maximal invariant. So if $h_{T}$ is of the form $h_{T}=h_{T}^{\phi} \circ \phi_{T}$, then Theorem 1 applies and yields an asymptotic efficiency statement among all invariant tests in $\mathfrak{C}$.

UnIT RoOT TEST EXAMPLE-CONTINUED: Consider the problem of testing for a unit root in a model with unknown mean, and suppose $\omega=1$ is known for simplicity. A maximal invariant is given by the demeaned data $\left\{\hat{u}_{T, t}\right\}_{t=1}^{T}$ with $\hat{u}_{T, t}=y_{T, t}-\bar{y}_{T}$, where $\bar{y}_{T}=T^{-1} \sum_{t=1}^{T} y_{T, t}$ and $Y_{T}=\left(y_{T, 1}, \ldots, y_{T, T}\right)^{\prime}$. The typical model satisfies $T^{-1 / 2} \hat{u}_{T,\lfloor\cdot T\rfloor} \rightsquigarrow J_{c}^{\mu}(\cdot)$, where $J_{c}^{\mu}(s)=J_{c}(s)-\int_{0}^{1} J_{c}(l) d l$. Theorem 1 now shows that rejecting for large values of $L^{\mu}\left(T^{-1 / 2} \hat{u}_{T, \downarrow \cdot T\rfloor}\right)$ is asymptotically point-optimal among all tests whose asymptotic null rejection probability 
is at most $\alpha$ whenever $T^{-1 / 2} \hat{u}_{T, \backslash \cdot T\rfloor} \rightsquigarrow J_{c}^{\mu}(\cdot)$, where $L^{\mu}$ is the Radon-Nikodym derivative of the probability measure of $J_{c_{1}}^{\mu}$ with respect to the measure of $J_{0}^{\mu}{ }^{4}$ Rejecting for large values of $L^{\mu}\left(T^{-1 / 2} \hat{u}_{T, \downarrow-T\rfloor}\right)$ is, therefore, the asymptotically point-optimal translation invariant test in the class of tests that do not overreject asymptotically whenever $T^{-1 / 2} \hat{u}_{T, \downarrow \cdot T\rfloor} \rightsquigarrow J_{0}^{\mu}(\cdot)$.

In the second case, one considers the typical weak convergence in a model with nuisance parameters, and applies invariance only in the limiting problem. Formally, let $\tilde{g}: R \times S \mapsto S$ be such that the $S \mapsto S$ functions $x \mapsto \tilde{g}(r, x)$, indexed by $r \in R$, form the group $\tilde{\mathcal{G}}$. Further suppose $\tilde{\phi}: S \mapsto S$ is a maximal invariant to $\tilde{\mathcal{G}}$, so that the efficient invariant test in the limiting problem $\varphi_{S}^{\phi *}$ is the efficient test of $H_{0}^{\phi, l_{p}}: \tilde{X} \sim \tilde{\phi} P(\theta), \theta \in \Theta_{0}$ against $H_{1}^{\phi, l p}: \tilde{X} \sim \tilde{\phi} P(\theta)$, $\theta \in \Theta_{1}$, where $\tilde{X}=\tilde{\phi}(X)$. It is not clear whether or in which sense this test, evaluated at sample analogues would be asymptotically efficient.

UNIT RoOT TEST EXAMPLE-CONTINUED: In the parametrization $y_{T, t}=$ $u_{T, t}+T^{1 / 2} \alpha_{y}$, we obtain with $\theta=\left(c, \alpha_{y}\right)^{\prime}$ that $T^{-1 / 2} y_{T, \downarrow \cdot T\rfloor} \rightsquigarrow J_{\theta}^{y}(\cdot)$, where $J_{\theta}^{y}(s)=J_{c}(s)+\alpha_{y}$. The parameter $\alpha_{y}$ is a nuisance parameters in the limiting problem. Define the transformations $\tilde{g}: \mathbb{R} \times D_{[0,1]} \mapsto D_{[0,1]}$ as $\tilde{g}(r, x)=x(\cdot)+r$, with $r \in R=\mathbb{R}$. The limiting problem is invariant to these transformations and $\tilde{\phi}: D_{[0,1]} \mapsto D_{[0,1]}$ with $\tilde{\phi}(x)=x(\cdot)-\int_{0}^{1} x(l) d l$ is a maximal invariant. Since $\tilde{\phi}\left(J_{\theta}^{y}\right) \sim J_{c}^{\mu}(\cdot)$, the point-optimal invariant test in the limiting problem rejects for large values of $L^{\mu} \circ \tilde{\phi}\left(J_{\theta}^{y}\right)$. This test, evaluated at sample analogues, yields $L^{\mu}\left(T^{-1 / 2} \hat{u}_{T, \mid \cdot T\rfloor}\right)$, just as above.

Even though in this example, the efficient invariant test in the limiting problem, evaluated at sample analogues, is small sample invariant, one still cannot claim this test to be the asymptotically point-optimal test among all small sample invariant tests in the class $\mathfrak{C}$ relative to the weak convergence $T^{-1 / 2} y_{T, \downarrow \cdot T\rfloor} \rightsquigarrow$ $J_{\theta}^{y}(\cdot)$. The reason is that the set of models satisfying $T^{-1 / 2} y_{T, \downarrow \cdot T\rfloor} \rightsquigarrow J_{\theta}^{y}(\cdot)$ is a proper subset of the set of models satisfying $T^{-1 / 2} \hat{u}_{T, \backslash \cdot T\rfloor} \rightsquigarrow J_{c}^{\mu}(\cdot)$. The efficiency of $L^{\mu}\left(T^{-1 / 2} \hat{u}_{T, \mid \cdot T\rfloor}\right)$ noted above is thus relative to a potentially smaller class of tests, and it remains unclear whether an efficiency claim can also be made relative to the weaker constraint of asymptotic size control whenever $T^{-1 / 2} y_{T, \backslash \cdot T\rfloor} \rightsquigarrow J_{\theta}^{y}(\cdot)$.

We now show how one can make an asymptotic efficiency claim when invariance is employed in the limiting problem by relating the limiting group of trans-

\footnotetext{
${ }^{4}$ Under the assumption of $u_{T, 0}=0$ for the initial condition, $L^{\mu}(x)=L(x(\cdot)-x(0))$, where $L$ is the Radon-Nikodym derivative of the probability measure of $J_{\theta_{1}}$ with respect to the measure of $J_{0}$, so that rejecting for large values of $L^{\mu}\left(T^{-1 / 2} \hat{u}_{T, \backslash \cdot T\rfloor}\right)$ leads to the same asymptotic power as without translation invariance. This equivalence does not hold, however, when the initial condition $u_{T, 0}$ is on the same order of magnitude as $u_{T,\lfloor s T\rfloor}$ for $s>0$. See Müller and Elliott (2003) for discussion.
} 
formations $\tilde{g}$ to a sequence of small sample groups. So for each $T$, suppose the measurable function $g_{T}: R \times \mathbb{R}^{n T} \mapsto \mathbb{R}^{n T}$ is such that the $\mathbb{R}^{n T} \mapsto \mathbb{R}^{n T}$ functions $y \mapsto g_{T}(r, y)$, indexed by $r \in R$, form the group $\mathcal{G}_{T}$. Let $\phi_{T}: \mathbb{R}^{n T} \mapsto \mathbb{R}^{n T}$ be a maximal invariant of $\mathcal{G}_{T}$. Assume that the small sample and limiting invariance correspond in the sense that the small sample maximal invariant converges weakly to the maximal invariant of the limiting problem, that is,

$$
\left(h_{T} \circ \phi_{T}\right) F_{T}(m, \theta) \rightsquigarrow \tilde{\phi} P(\theta) \quad \text { pointwise for } \theta \in \Theta .
$$

Let $\mathcal{M}^{\phi}$ be the set of models $m$ satisfying (16). Since $\varphi_{S}^{\phi *}$ was assumed to be the efficient test of $H_{0}^{\phi, l p}: \tilde{X} \sim \tilde{\phi} P(\theta), \theta \in \Theta_{0}$, against $H_{1}^{\phi, l p}: \tilde{X} \sim \tilde{\phi} P(\theta), \theta \in \Theta_{1}$, one can apply Theorem 1 to conclude that $\varphi_{S}^{\phi *} \circ h_{T} \circ \phi_{T}$ is the asymptotically efficient small sample invariant (with respect to $\mathcal{G}_{T}$ ) test in the class of tests that are asymptotically valid for all models $\mathcal{M}^{\phi}$. As noted in the unit root example above, however, one cannot conclude that $\varphi_{S}^{\phi *} \circ h_{T} \circ \phi_{T}$ is also the asymptotically efficient small sample invariant test in the (potentially larger) class of tests that are asymptotically valid for all models in $\mathcal{M}$, that is, relative to the weak convergence $h_{T} F_{T}(m, \theta) \rightsquigarrow P(\theta)$.

What could go wrong is that for some small sample invariant test, (8), (10), and (11) hold for all $m \in \mathcal{M}$, but there exists $m^{\prime} \in \mathcal{M}^{\phi}$ for which at least one of (8), (10), and (11) does not hold. But this cannot happen if for any $m^{\prime} \in \mathcal{M}^{\phi}$, there exists a corresponding $m \in \mathcal{M}$ such that $\phi_{T} F_{T}(m, \theta)=F_{T}\left(m^{\prime}, \theta\right)$. Given that the constraints (8), (10), and (11) are pointwise in $\theta$, the following theorem shows this to be the case under suitable conditions.

THEOREM 2: Pick any $m \in \mathcal{M}^{\phi}$ and $\theta \in \Theta$. Suppose (i) $R$ is a separable and complete metric space; (ii) the mapping $x \mapsto \tilde{g}(r, x)$ is continuous for all $r \in R$; (iii) there exists a measurable function $\tilde{\rho}: S \mapsto R$ such that $x=\tilde{g}(\tilde{\rho}(x), \tilde{\phi}(x))$ for all $x \in S$; (iv) for all $r \in R, d_{S}\left(h_{T}\left(g_{T}\left(r, \phi_{T}\left(Y_{T}\right)\right)\right), \tilde{g}\left(r, h_{T}\left(\phi_{T}\left(Y_{T}\right)\right)\right)\right) \rightsquigarrow 0$ when $Y_{T} \sim F_{T}(m, \theta)$, where $d_{S}$ is the metric on $S ;(v) \tilde{\phi}$ and $\phi_{T}$ select specific orbits, that is, for all $y \in \mathbb{R}^{n T}$ and $x \in S$, there exist $r_{y}, r_{x} \in R$ so that $\phi_{T}(y)=g_{T}\left(r_{y}, y\right)$ and $\tilde{\phi}(x)=\tilde{g}\left(r_{x}, x\right)$. Then there exists a sequence of measures $G_{T}$ on $\mathfrak{B}\left(\mathbb{R}^{n T}\right)$ such that $\phi_{T} F_{T}(m, \theta)=\phi_{T} G_{T}(\theta)$ and $h_{T} G_{T}(\theta) \rightsquigarrow P(\theta)$.

UNIT RoOT TEST EXAMPLE-CONTINUED: With $g_{T}\left(r, Y_{T}\right)=\left(y_{T, 1}+r T^{1 / 2}\right.$, $\left.\ldots, y_{T, T}+r T^{1 / 2}\right)^{\prime}, \phi_{T}\left(Y_{T}\right)=g_{T}\left(-T^{-1 / 2} \bar{y}_{T}, Y_{T}\right)$, and $\tilde{\rho}(x)=\int_{0}^{1} x(s) d s$, we find $\left(h_{T} \circ \phi_{T}\right)\left(Y_{T}\right)=T^{-1 / 2} \hat{u}_{T,\lfloor\cdot T\rfloor} \rightsquigarrow J_{c}^{\mu} \sim \tilde{\phi}\left(J_{c}\right), \sup _{y \in \mathbb{R}^{T}} d_{D_{[0,1]}}\left(T^{-1 / 2}\left(y_{\lfloor s T\rfloor}+\right.\right.$ $\left.\left.T^{1 / 2} r\right), T^{-1 / 2} y_{\lfloor s T\rfloor}+r\right)=0$, and $x=\tilde{\phi}(x)+\tilde{\rho}(x)=\tilde{g}(\tilde{\rho}(x), \tilde{\phi}(x))$ for all $x \in$ $D_{[0,1]}$, so that the assumptions of Theorem 2 hold. We can, therefore, conclude that rejecting for large values of $L^{\mu}\left(T^{-1 / 2} \hat{u}_{T, \downarrow \cdot T]}\right)$ is also the asymptotically point-optimal test among all translation invariant tests with asymptotic null rejection probability of at most $\alpha$ whenever $T^{1 / 2} y_{T,\lfloor\cdot T\rfloor} \rightsquigarrow J_{0}(\cdot)+\alpha_{y}, \alpha_{y} \in \mathbb{R}$. 
For the proof of Theorem 2, note that with $x=\tilde{g}(\tilde{\rho}(x), \tilde{\phi}(x))$ for all $x \in S$, one can construct the distribution $P(\theta)$ by applying an appropriate random transformation $\tilde{g}$ to each $x$ drawn under $\tilde{\phi} P(\theta)$. Under the assumptions of Theorem 2, for each $F_{T}(m, \theta)$ with $m \in \mathcal{M}^{\phi}$ and $\theta \in \Theta$, one can thus obtain a corresponding data generating process $G_{T}$ with $h_{T} G_{T}(\theta) \rightsquigarrow P(\theta)$ by applying an appropriate random transformation $g_{T} \in \mathcal{G}_{T}$ for each $T$.

While asymptotic efficiency statements about invariant tests based on Theorems 1 and 2 require a tight link between the limiting group $\tilde{\mathcal{G}}$ and the small sample groups $\mathcal{G}_{T}$, the link does not need to be perfect: Even if the distribution of the limiting maximal invariant $\tilde{\phi} P(\theta)$ does not depend on a subset of the parameter $\theta$ (so that a nuisance parameter is eliminated by invariance), it is not assumed that the small sample counterpart $\phi_{T} F_{T}(m, \theta)$ shares this feature. Also assumption (iv) does not require the small sample and limiting group actions to exactly coincide, which is useful for, say, arguing for the asymptotic efficiency of translation and trend invariant unit root tests with respect to the weak convergence $T^{-1 / 2} y_{T,\lfloor\cdot T\rfloor} \rightsquigarrow J_{c}(\cdot)+\alpha_{y}+\cdot \beta_{y}$.

\subsection{Uniformity}

The discussion so far concerned the pointwise asymptotic properties of tests $\varphi_{T}$, that is, the rejection probability as $T \rightarrow \infty$ for a fixed model $m$ and parameter value $\theta \in \Theta$. As argued by Dufour (1997) and Pötscher (2002), among others, one might instead want to focus on uniform properties of tests in $m$ and in $\theta \in \Theta$.

It is clear, however, that the pointwise constraint (8) is not enough to generate any useful bound for the small sample rejection probabilities of tests, even pointwise in $\theta$. The reason is that for any fixed sample size $T$, there exist models in $\mathcal{M}$ for which the distribution of $Y_{T}$ is arbitrary, since the convergence $P_{T}(m, \theta)=h_{T} F_{T}(m, \theta) \rightsquigarrow P(\theta)$ occurs "later." The interest in results derived from (2) must stem from an implicit assumption that these asymptotic properties are reasonable approximations for the small sample distribution of $X_{T}$ in the actual sample size $T$.

A formalization of this implicit assumption is obtained by imposing a lower limit on the speed of convergence. For two probability measures $\mu_{1}$ and $\mu_{2}$ on $\mathfrak{B}(S)$, define $\Delta_{B L}$ as $\Delta_{B L}\left(\mu_{1}, \mu_{2}\right)=\sup _{\|f\|_{B L} \leq 1}\left|\int f d \mu_{1}-\int f d \mu_{2}\right|$, where $f: S \mapsto \mathbb{R}$ are $\mathfrak{B}(S) \backslash \mathfrak{B}(\mathbb{R})$ measurable and $\|f\|_{B L}=\sup _{x \in S}|f(x)|+$ $\sup _{x \neq y, x, y \in S} \frac{|f(x)-f(y)|}{d_{S}(x, y)}$. It is known that $\Delta_{B L}$ metrizes weak convergence on separable metric spaces (Dudley (2002, p. 395)). Also, let the real sequence $\delta=\left\{\delta_{T}\right\}_{T=1}^{\infty}$ be such that $\delta_{T} \rightarrow 0$. Now define $\mathcal{M}^{u}(\delta)$ ( $u$ denotes uniform) as the set of models $m$ satisfying

$$
\sup _{\theta \in \Theta} \Delta_{B L}\left(P_{T}(m, \theta), P(\theta)\right) \leq \delta_{T},
$$


that is, $\mathcal{M}^{u}(\delta)$ is the collection of models $m$ for which the distribution $P_{T}(m, \theta)=h_{T} F_{T}(m, \theta)$ of $h_{T}\left(Y_{T}\right)$ differs by at most $\delta_{T}$ from its limit $P(\theta)$ as measured by $\Delta_{B L}$, uniformly over $\Theta$. It then makes sense to ask whether the rejection probability of a test $\varphi_{T}$ converges to the nominal level uniformly over $\theta \in \Theta_{0}$ and $\mathcal{M}^{u}(\delta)$, that is, if

$$
\underset{T \rightarrow \infty}{\limsup } \sup _{\theta \in \Theta_{0}, m \in \mathcal{M}^{u}(\delta)} \int \varphi_{T} d F_{T}(m, \theta) \leq \alpha .
$$

By the continuity of $\varphi_{S}^{*},(18)$ holds for the test $\varphi_{T}=\hat{\varphi}_{T}^{*}$ in Theorem 1 , that is, for large enough $T$, the rejection probability of $\hat{\varphi}_{T}^{*}$ is close to $\alpha$ for all models in $\mathcal{M}^{u}(\delta)$, uniformly over $\Theta_{0} .{ }^{5}$ Similar restrictions and arguments could be made regarding the constraints (10) and (11).

It is not clear, however, whether all tests in the class that satisfy (8) also satisfy (18) or vice versa. Theorem 1 , therefore, does not imply that $\hat{\varphi}_{T}^{*}$ also maximizes asymptotic weighted average power in the class of all tests that satisfy (18). A partial result in that regard is provided by the following theorem for the case of a single null hypothesis and without constraints (10) and (11).

THEOREM 3: Suppose $\Theta_{0}=\left\{\theta_{0}\right\}$, let $L: \Theta \times S \mapsto \mathbb{R}$ be the Radon-Nikodym derivative of $P(\theta)$ with respect to $P\left(\theta_{0}\right)$, define $\bar{L}(x)=\int L(\theta, x) d w(\theta)$, let $\varphi_{S}^{*}: S \mapsto[0,1]$ be the level $\alpha$ test that rejects for large values of $\bar{L}$, and define $\hat{\varphi}_{T}^{*}=\varphi_{S}^{*} \circ h_{T}$. Suppose that for all $\varepsilon>0$ there exists an open set $D_{\varepsilon} \in \mathfrak{B}(S)$ with $\int_{D_{\varepsilon}} d P\left(\theta_{0}\right)>1-\varepsilon$ so that the $D_{\varepsilon} \mapsto \mathbb{R}$ function $x \mapsto \bar{L}(x)$ is Lipschitz, suppose there exists a model $m_{0}$ with $\Delta_{B L}\left(P_{T}\left(m_{0}, \theta_{0}\right), P\left(\theta_{0}\right)\right) / \delta_{T} \rightarrow 0$, and assume that $\Delta_{B L}\left(\int P_{T}\left(m_{1}, \theta\right) d w(\theta), \int P(\theta) d w(\theta)\right) / \delta_{T} \rightarrow 0$. Then for any test $\varphi_{T}$ that satisfies (18), $\lim \sup _{T \rightarrow \infty} \operatorname{WAP}\left(\varphi_{T}, m_{1}\right) \leq \lim _{T \rightarrow \infty} \operatorname{WAP}\left(\hat{\varphi}_{T}^{*}, m_{1}\right)=\operatorname{WAP}\left(\varphi_{S}^{*}\right)$.

Under a stronger continuity assumption on the limiting problem, Theorem 3 shows that no test can satisfy (18) and have higher asymptotic weighted average power than $\hat{\varphi}_{T}^{*}$ in models whose (average) weak convergence under the alternative is faster than the lower bound $\delta_{T}$. The proof closely follows the heuristic sketch of the proof of Theorem 1 outlined at the end of Section 2.4 and exploits the linearity in both the definition of $\Delta_{B L}$ and the probability assignments in the tilted model $G_{T}$. Dudley (2002, p. 411) showed that the Prohorov metric $\Delta_{P}$ (which also metrizes weak convergence) satisfies $\Delta_{P} \leq 2 \Delta_{B L}^{1 / 2}$ and $\Delta_{B L} \leq 2 \Delta_{P}$, so that Theorem 3 could equivalently be formulated in terms of $\Delta_{P}$.

\footnotetext{
${ }^{5}$ Construct a sequence of functions $\ell_{T}: S \mapsto \mathbb{R}$ with $\ell_{T}(x) \geq \varphi_{S}^{*}(x)$ for $x \in S$ and $\delta_{T}\left\|\ell_{T}\right\|_{B L} \rightarrow$ 0 that converge to $\varphi_{S}^{*}$ pointwise $\mu_{P}$ almost everywhere, as in Chapter 7.1 of Pollard (2002). Then $\sup _{\theta \in \Theta_{0}, m \in \mathcal{M}^{u}(\delta)} \int \hat{\varphi}_{T}^{*} d F_{T}(m, \theta)-\alpha \leq \sup _{\theta \in \Theta_{0}, m \in \mathcal{M}^{u}(\delta)} \int \ell_{T}\left(d P_{T}(m, \theta)-d P(\theta)\right) \leq$ $\left\|\ell_{T}\right\|_{B L} \sup _{\theta \in \Theta_{0}} \Delta_{B L}\left(P_{T}(m, \theta), P(\theta)\right) \rightarrow 0$, where the last inequality uses $\left\|f_{1} \cdot f_{2}\right\|_{B L} \leq\left\|f_{1}\right\|_{B L}$. $\left\|f_{2}\right\|_{B L}$. For this uniform validity result, it would suffice to assume (17) with $\Theta$ replaced by $\Theta_{0}$.
} 


\section{APPLICATIONS}

\subsection{Unit Root Tests With Stationary Covariates}

Elliott and Jansson (2003) considered the model

$$
\left(\begin{array}{c}
y_{T, t} \\
x_{T, t}
\end{array}\right)=\left(\begin{array}{c}
\alpha_{y}+\beta_{y} t \\
\alpha_{x}+\beta_{x} t
\end{array}\right)+\left(\begin{array}{c}
u_{T, t} \\
\nu_{T, t}^{x}
\end{array}\right)
$$

where $Y_{T}=\left(\left(y_{T, 1}, x_{T, 1}^{\prime}\right)^{\prime}, \ldots,\left(y_{T, T}, x_{T, T}^{\prime}\right)\right)^{\prime} \in \mathbb{R}^{n T}$ is observed; $\alpha_{y}, \beta_{y}$, and $u_{T, t}=$ $\rho_{T} u_{T, t-1}+\nu_{T, t}^{y}$ are scalars, $u_{T, 0}=O_{p}(1), \rho_{T}=1-c / T$ for some fixed $c \geq 0$, and $x_{T, t}, \alpha_{x}, \beta_{x}$, and $\nu_{T, t}^{x}$ are $n-1$ dimensional vectors. The objective is to efficiently exploit the stationary covariates $x_{T, t}$ in the construction of a test of the null hypothesis of a unit root in $y_{T, t}, H_{0}: c=0$ against the alternative $H_{1}: c>0$. Consider first the case with $\alpha_{y}=\alpha_{x}=\beta_{y}=\beta_{x}=0$ known. The approach of Elliott and Jansson (2003) is first to apply the Neyman-Pearson lemma to determine, for each $T$, the point-optimal test against $c=c_{1}$ when $\nu_{T, t}=\left(\nu_{T, t}^{y}, \nu_{T, t}^{x \prime}\right)^{\prime} \sim$ i.i.d. $\mathcal{N}(0, \Omega)$ for known $\Omega$. In a second step, they constructed a feasible test that is (i) asymptotically equivalent to the point-optimal test when $\nu_{T, t} \sim$ i.i.d. $\mathcal{N}(0, \Omega)$ and (ii) that is robust to a range of autocorrelation structures and error distributions.

To apply the results in Sections 2 and 3 of this paper, we consider the typical weak convergence properties of model (19). Standard weak dependence assumptions on $\nu_{T, t}$ imply for some suitable long-run covariance matrix estimator $\hat{\Omega}_{T}$ that

$$
\hat{\Omega}_{T} \stackrel{p}{\rightarrow} \Omega \quad \text { and } \quad G_{T}(\cdot)=\left(\begin{array}{c}
T^{-1 / 2} u_{T, \downarrow \cdot T\rfloor} \\
T^{-1 / 2} \sum_{t=1}^{\lfloor\cdot T\rfloor} \nu_{T, t}^{x}
\end{array}\right) \rightsquigarrow G(\cdot),
$$

where $\Omega$ is positive definite, $G(s)=\int_{0}^{s} \operatorname{diag}\left(e^{-c(s-r)}, 1, \ldots, 1\right) \Omega^{1 / 2} d W(r)$, and $W$ is a $n \times 1$ standard Wiener process. By Girsanov's theorem, the RadonNikodym derivative of the distribution of $G$ with $c=c_{1}$ with respect to the distribution of $G$ with $c=0$, evaluated at $G=\left(G_{y}, G_{x}^{\prime}\right)^{\prime}$, is given by

$$
\begin{aligned}
L(\Omega, G)= & \exp \left[-c_{1} \int_{0}^{1} G(s)^{\prime} S_{1} \Omega^{-1} d G(s)\right. \\
& \left.-\frac{1}{2} c_{1}^{2} \int_{0}^{1} G(s)^{\prime} S_{1} \Omega^{-1} S_{1} G(s) d s\right] \\
= & \exp \left[-\frac{1}{2} c_{1} \omega_{y y}\left(G_{y}(1)^{2}-\sigma_{y y}^{2}\right)-c_{1} \omega_{y x} \int_{0}^{1} G_{y}(s) d G_{x}(s)\right. \\
& \left.-\frac{1}{2} c_{1}^{2} \omega_{y y} \int_{0}^{1} G_{y}(s)^{2} d s\right],
\end{aligned}
$$


where $S_{1}$ is the $n \times n$ matrix $S_{1}=\operatorname{diag}(1,0, \ldots, 0), \sigma_{y y}^{2}$ is the $(1,1)$ element of $\Omega$, and the first row of $\Omega^{-1}$ is $\left(\omega_{y y}, \omega_{y x}\right)$. By the Neyman-Pearson lemma, the point-optimal test in the limiting problem rejects for large values of $L(\Omega, G)$. Since $\int_{0}^{1} G_{y}(s) d G_{x}(s)$ is not a continuous mapping, we cannot directly apply Theorem 1. However, typical weak dependence assumptions on $\nu_{T, t}$ also imply (see, for instance, Phillips (1988), Hansen (1990), and de Jong and Davidson (2000)) that

$$
Y_{T}=T^{-1} \sum_{t=2}^{T} u_{T, t-1} \nu_{T, t}^{x}-\hat{\Sigma}_{T} \rightsquigarrow Y=\int_{0}^{1} G_{y}(s) d G_{x}(s)
$$

for a suitably defined $(n-1) \times 1$ vector $\hat{\Sigma}_{T} \stackrel{p}{\rightarrow} \Sigma$ (which equals $\sum_{s=1}^{\infty} E\left[\nu_{T, t}^{y} \nu_{T, t+s}^{x}\right]$ when $\nu_{T, t}$ is covariance stationary) jointly with (20). Clearly, the RadonNikodym derivative of the measure of $(G, Y)$ for $c=c_{1}$ with respect to the measure of $(G, Y)$ with $c=0$, evaluated at $G$, is also given by $L(\Omega, G)$ in (21), and one can write $L(\Omega, G)=L^{Y}(\Omega, G, Y)$ for a continuous function $L^{Y}$. The discussion of Section 3.1 thus applies, and Theorem 1 shows that rejecting for large values of $L^{\gamma}\left(\hat{\Omega}_{T}, G_{T}, Y_{T}\right)$ is the point-optimal unit root test for the alternative $c=c_{1}$ in the class of tests that have correct asymptotic null rejection probabilities whenever (20) and (22) hold.

Since the model with $\nu_{T, t} \sim$ i.i.d. $\mathcal{N}(0, \Omega)$ satisfies (20) and (22), the test derived by Elliott and Jansson (2003) is by construction-as explained in Comment 1 in Section 2.5-asymptotically equivalent to a test that rejects for large values of $L^{Y}\left(\hat{\Omega}_{T}, G_{T}, Y_{T}\right)$. The derivation here, which starts with the RadonNikodym derivative directly, is arguably a more straightforward way to determine a test in this equivalence class. Furthermore, while Elliott and Jansson (2003) can only claim optimality for the model with i.i.d. Gaussian disturbances, Theorem 1 shows that the test is efficient against all alternatives satisfying (20) and (22) with $c=c_{1}$ if one imposes size control for all models satisfying (20) and (22) with $c=0$. In other words, under this constraint, no test exists with higher asymptotic power for any disturbance distribution or autocorrelation structure satisfying (20) and (22) with $c=c_{1}$.

When the deterministic terms are not fully known, that is, the parameters $\alpha_{y}$, $\alpha_{x}, \beta_{y}$, and/or $\beta_{x}$ are not known, it is natural to impose appropriate invariance requirements. Specifically, for the case where $\alpha_{y}$ and $\alpha_{x}$ are unconstrained and $\beta_{y}=\beta_{x}=0$, consider

$$
\left\{\left(y_{T, t}, x_{T, t}^{\prime}\right)^{\prime}\right\}_{t=1}^{T} \rightarrow\left\{\left(y_{T, t}+a_{y}, x_{T, t}^{\prime}+a_{x}^{\prime}\right)^{\prime}\right\}_{t=1}^{T}, \quad a_{y} \in \mathbb{R}, a_{x} \in \mathbb{R}^{n-1} .
$$

A maximal invariant of this group of transformations is given by the demeaned data $\left\{\left(\hat{y}_{T, t}, \hat{x}_{T, t}^{\prime}\right)^{\prime}\right\}_{t=1}^{T}$, where $\hat{y}_{T, t}=y_{T, t}-\bar{y}_{T}, \hat{x}_{T, t}=x_{T, t}-\bar{x}_{T}, \bar{y}_{T}=T^{-1} \sum_{t=1}^{T} y_{T, t}$, and $\bar{x}_{T}=T^{-1} \sum_{t=1}^{T} x_{T, t}$. Elliott and Jansson (2003) derived the limiting behavior of the likelihood ratio statistics of this maximal invariant when $\nu_{T, t} \sim$ 
i.i.d. $\mathcal{N}(0, \Omega)$, and thus obtained the asymptotically point-optimal invariant unit root test under that assumption. Considering again the weak convergence properties of a typical model, we obtain

$$
\begin{aligned}
& \left(\begin{array}{c}
T^{-1 / 2} \hat{y}_{T,\lfloor\cdot T\rfloor} \\
T^{-1 / 2} \sum_{t=1}^{\lfloor\cdot T\rfloor} \hat{x}_{T, t} \\
T^{-1 / 2} \sum_{t=2}^{T} \hat{y}_{T, t-1} \hat{x}_{T, t}-\hat{\Sigma}_{T}
\end{array}\right) \rightsquigarrow\left(\begin{array}{c}
\hat{G}_{y}(\cdot) \\
\hat{G}_{x}(\cdot) \\
\int_{0}^{1} \hat{G}_{y}(s) d \hat{G}_{x}(s)
\end{array}\right) \quad \text { and } \\
& \hat{\Omega}_{T} \stackrel{p}{\rightarrow} \Omega,
\end{aligned}
$$

where $\hat{G}_{y}(s)=G_{y}(s)-\int_{0}^{1} G_{y}(l) d l$ and $\hat{G}_{x}(s)=G_{x}(s)-s G_{x}(1)$, and $\hat{\Omega}_{T}$ and $\hat{\Sigma}_{T}$ are functions of $\left\{\left(\hat{y}_{T, t}, \hat{x}_{T, t}^{\prime}\right)^{\prime}\right\}_{t=1}^{T}$. For brevity, we omit an explicit expression for the Radon-Nikodym derivative $L^{\hat{G}}$ of the measure of $\left(\hat{G}_{y}, \hat{G}_{x}\right)$ with $c=c_{1}$ with respect to the measure of $\left(\hat{G}_{y}, \hat{G}_{x}\right)$ when $c=0$. By the Neyman-Pearson lemma and Theorem 1, rejecting for large values of $L^{\hat{G}}$, evaluated at sample analogues, is the asymptotically point-optimal test in the class of tests that are asymptotically valid whenever (24) holds with $c=0$.

Furthermore, in the notation of Section 3.2, with $h_{T}\left(Y_{T}\right)=\left(T^{-1 / 2} y_{T,\lfloor\cdot T\rfloor}\right.$, $\left.T^{-1 / 2} \sum_{t=1}^{\lfloor\cdot T\rfloor} x_{T, t}, T^{-1} \sum_{t=2}^{T} y_{T, t-1} x_{T, t}-\hat{\Sigma}_{T}\right) \in D_{[0,1]} \times D_{[0,1]}^{n-1} \times \mathbb{R}^{n-1}, r=\left(r_{y}, r_{x}^{\prime}\right)^{\prime} \in$ $\mathbb{R} \times \mathbb{R}^{n-1}, g_{T}\left(r,\left\{\left(y_{T, t}, x_{T, t}^{\prime}\right)^{\prime}\right\}_{t=1}^{T}\right)=\left\{\left(y_{T, t}+T^{1 / 2} r_{y}, x_{T, t}^{\prime}+T^{-1 / 2} r_{x}^{\prime}\right)^{\prime}\right\}_{t=1}^{T}, \tilde{g}(r,(y, x$, $z))=\left(y(\cdot)+r_{y}, x(\cdot)+r_{x} \cdot, z+r_{y}(x(1)-x(0))+r_{x} \int_{0}^{1} y(s) d s+r_{y} r_{x}\right), \phi_{T}\left(\left\{\left(y_{T, t}\right.\right.\right.$, $\left.\left.\left.x_{T, t}^{\prime}\right)^{\prime}\right\}_{t=1}^{T}\right)=\left\{\left(\hat{y}_{T, t}, \hat{x}_{T, t}^{\prime}\right)^{\prime}\right\}_{t=1}^{T}$, and $\tilde{\phi}(y, x, z)=\left(y(\cdot)-\int_{0}^{1} y(s) d s, x(\cdot)-\cdot x(1)\right.$, $z-(x(1)-x(0)) \int_{0}^{1} y(s) d s$ ), we find that Theorem 2 (and the discussion in Section 3.1) is applicable and that rejecting for large values of $L^{\hat{G}}$, evaluated at sample analogues, is also the small sample invariant, asymptotically pointoptimal unit root test in the class of tests that are asymptotically valid whenever

$$
\begin{aligned}
& \left(\begin{array}{c}
T^{-1 / 2} y_{T,\lfloor\cdot T\rfloor} \\
T^{-1 / 2} \sum_{t=1}^{\lfloor\cdot T\rfloor} x_{T, t} \\
T^{-1 / 2} \sum_{t=2}^{T} y_{T, t-1} x_{T, t}-\hat{\Sigma}_{T}
\end{array}\right) \rightsquigarrow\left(\begin{array}{c}
G_{y}^{\alpha}(\cdot) \\
G_{x}^{\alpha}(\cdot) \\
\int_{0}^{1} G_{y}^{\alpha}(s) d G_{x}^{\alpha}(s)
\end{array}\right) \text { and } \\
& \hat{\Omega}_{T} \stackrel{p}{\rightarrow} \Omega,
\end{aligned}
$$

where $G_{y}^{\alpha}(s)=G_{y}(s)+\alpha_{y}$ and $G_{x}^{\alpha}(s)=G_{x}(s)+s \alpha_{x}$ for some $\alpha_{y} \in \mathbb{R}, \alpha_{x} \in \mathbb{R}^{n-1}$. 


\subsection{Linear Regression With Weak Instruments}

As in Andrews, Moreir, and Stock (2006) (subsequently abbreviated AMS), consider the problem of inference about the coefficient of a scalar endogenous variable in the presence of weak instruments. The reduced form equations are given by (cf. equation (2.4) of AMS)

$$
\begin{aligned}
& y_{1, t}=z_{t}^{\prime} \pi \beta+x_{t}^{\prime} \zeta_{1}+v_{t, 1}, \\
& y_{2, t}=z_{t}^{\prime} \pi+x_{t}^{\prime} \zeta_{2}+v_{t, 2}
\end{aligned}
$$

for $t=1, \ldots, T$, where $y_{1, t}$ and $y_{2, t}$ are scalars, $z_{t}$ is $k \times 1, x_{t}$ is $p \times 1$, and $z_{t}$ are the residuals of a linear regression of the original instruments $\tilde{z}_{t}$ on $x_{t}$. AMS initially considered small sample efficient tests of

$$
H_{0}: \beta=\beta_{0}
$$

for nonstochastic $\left(x_{t}, z_{t}\right)$ and $v_{t}=\left(v_{1, t}, v_{2, t}\right)^{\prime} \sim$ i.i.d. $\mathcal{N}(0, \Omega)$ with $\Omega$ known. A sufficiency argument shows that tests may be restricted to functions of the $2 k \times 1$ multivariate normal statistic

$$
\sum_{t=1}^{T}\left(\begin{array}{c}
z_{t} y_{1, t} \\
z_{t} y_{2, t}
\end{array}\right) \sim \mathcal{N}\left(\left(\begin{array}{c}
S_{z} \pi \beta \\
S_{z} \pi
\end{array}\right), \Omega \otimes S_{z}\right),
$$

where $S_{z}=\sum_{t=1}^{T} z_{t} z_{t}^{\prime}$ and AMS derived weighted average power maximizing similar tests that are invariant to the group of transformations

$$
\left\{z_{t}\right\}_{t=1}^{T} \rightarrow\left\{O z_{t}\right\}_{t=1}^{T} \text { for any orthogonal matrix } O .
$$

For their asymptotic analysis, AMS employed Staiger and Stock's (1997) weak instrument asymptotics, where $\pi=T^{-1 / 2} C$ for some fixed matrix $C$. AMS then exploited their small sample efficiency results to construct tests (i) that maximize weighted average asymptotic power among all asymptotically invariant and asymptotically similar test when $v_{t} \sim$ i.i.d. $\mathcal{N}(0, \Omega)$ independent of $\left\{\left(x_{t}^{\prime}, z_{t}^{\prime}\right)\right\}_{t=1}^{T}$ and (ii) that yield correct asymptotic null rejection probability under much broader conditions (the working paper of AMS contains the details of the construction of heteroskedasticity, and of heteroskedasticity and autocorrelation robust tests).

To apply the results from Sections 2 and 3 to this problem, consider the set of weak convergences in the double array version of model (25),

$$
\begin{aligned}
& \hat{D}_{Z}=T^{-1} \sum_{t=1}^{T} z_{T, t} z_{T, t}^{\prime} \stackrel{p}{\rightarrow} D_{z}, \quad \hat{\Sigma} \stackrel{p}{\rightarrow} \Sigma, \\
& X_{T}=T^{-1 / 2} \sum_{t=1}^{T}\left(\begin{array}{c}
z_{T, t} y_{1, T, t} \\
z_{T, t} y_{2, T, t}
\end{array}\right) \rightsquigarrow X \sim \mathcal{N}\left(\left(\begin{array}{c}
D_{z} C \beta \\
D_{z} C
\end{array}\right), \Sigma\right),
\end{aligned}
$$


where $\hat{\Sigma}$ is some estimator of the long-run variance of $\operatorname{vec}\left(z_{t} v_{t}^{\prime}\right)$, and $D_{z}$ and $\Sigma$ have full rank. The limiting problem in the sense of Section 2.2 above is thus the test of (26) based on observing the random variable $X$ distributed as in (29), with $D_{z}$ and $\Sigma$ known.

For $k=1$, that is, in the just identified case, this problem is exactly equivalent to the small sample problem (27) considered by AMS. In fact, already Moreira (2001) has shown that for $k=1$, the Anderson and Rubin (1949) statistic $\mathrm{AR}_{0}=\left(b_{0}^{\prime} X\right)^{2} / b_{0}^{\prime} \Sigma b_{0}$ with $b_{0}=\left(1,-\beta_{0}\right)^{\prime}$ yields the uniformly most powerful unbiased test $\varphi_{S}^{*}$. The discussion of Section 3.1 and Theorem 1 thus imply that for $k=1$, rejecting for large values of $\left(b_{0}^{\prime} X_{T}\right)^{2} / b_{0}^{\prime} \hat{\Sigma} b_{0}$ maximizes asymptotic power uniformly in all models that satisfy (29) with $\beta \neq \beta_{0}$ among all tests that are asymptotically unbiased with at most nominal asymptotic null rejection probability for all models that satisfy (29) with $\beta=\beta_{0}$.

The class of asymptotically valid tests for all models that satisfy (29) with $\beta=\beta_{0}$ is potentially quite small, since there are many ways $T^{-1 / 2} \sum_{t=1}^{T} z_{T, t} y_{T, t}$ can converge to a normal vector (for instance, the convergence is compatible with $z_{T, t} y_{T, t}=0$ for all $t<T / 2$ ). To the extent that one would be prepared to rule out such models a priori, this decreases the appeal of the efficiency result. But as discussed in Comment 7 in Section 2.5, one can impose additional weak convergences without necessarily affecting $\varphi_{s}^{*}$ : Supplementing (29) by the functional central limit theorem type convergence

$$
T^{-1 / 2} \sum_{t=1}^{\lfloor\cdot T\rfloor}\left(\begin{array}{c}
z_{T, t} y_{1, T, t} \\
z_{T, t} y_{2, T, t}
\end{array}\right) \rightsquigarrow G(\cdot)
$$

with

$$
G(s)=s\left(\begin{array}{c}
D_{z} C \beta \\
D_{z} C
\end{array}\right)+\Sigma^{1 / 2} W(s),
$$

where $W(s)$ is a $2 k \times 1$ standard Wiener process, for instance, rules out such pathological cases and yet still yields $\varphi_{S}^{*}$ to be the efficient test, since $G(1)$ is sufficient for the unknown parameters $C$ and $\beta$.

For $k>1$, Theorems 1 and 2 can again be invoked to yield analogous asymptotic efficiency statements for the statistics developed in AMS under the assumption that $\Sigma$ is of the Kronecker form $\Sigma=\Omega \otimes D_{z}$, as in (27). But this form naturally arises only in the context of a serially uncorrelated homoskedastic model, so the resulting efficiency statements are of limited appeal. The approach here thus points to a general solution of the limiting problem without the constraint $\Sigma=\Omega \otimes D_{z}$ as an interesting missing piece in the literature on efficient inference in linear regressions with weak instruments. 


\subsection{GMM Parameter Stability Tests}

Following Sowell (1996), suppose we are interested in testing the null hypothesis that a parameter $\beta \in \mathbb{R}^{k}$ in a GMM framework is constant through time. Parametrizing $\beta_{T, t}=\beta_{0}+T^{-1 / 2} \theta(t / T)$, where $\theta \in D_{[0,1]}^{k}$ and $\theta(0)$ is normalized to zero, $\theta(0)=0$, this is equivalent to the hypothesis test

$$
H_{0}: \theta=0 \text { against } H_{1}: \theta \neq 0 .
$$

Denote by $g_{T, t}(\beta) \in \mathbb{R}^{p}$ with $p \geq k$ the sample moment condition for $y_{T, t}$ evaluated at $\beta$, so that under the usual assumptions, the moment condition evaluated at the true parameter value satisfies a central limit theorem, that is, $T^{-1 / 2} \sum_{t=1}^{T} g_{T, t}\left(\beta_{T, t}\right) \rightsquigarrow \mathcal{N}(0, V)$ for some positive definite $p \times p$ matrix $V$. Furthermore, with $\hat{\beta}_{T}$ the usual full sample GMM estimator of $\beta$ with optimal weighting matrix converging to $V^{-1}$, we obtain under typical assumptions that for some suitable estimators $\hat{H}_{T}$ and $\hat{V}_{T}$ (cf. Theorem 1 of Sowell (1996)),

$$
G_{T}(\cdot)=T^{-1 / 2} \sum_{t=1}^{\llcorner\cdot T\rfloor} g_{T, t}\left(\hat{\beta}_{T}\right) \rightsquigarrow G(\cdot) \quad \text { and } \quad \hat{H}_{T} \stackrel{p}{\rightarrow} H, \quad \hat{V}_{T} \stackrel{p}{\rightarrow} V
$$

where the convergence to $G$ is on $D_{[0,1]}^{p}, G(s)=V^{1 / 2} W(s)-s H\left(H^{\prime} V^{-1} H\right)^{-1} \times$ $H^{\prime} V^{-1 / 2} W(1)+H\left(\int_{0}^{s} \theta(l) d l-s \int_{0}^{1} \theta(l) d l\right)$, where $W$ is a $p \times 1$ standard Wiener process and $H$ is some $p \times k$ full column rank matrix (which is the probability limit of the average of the partial derivatives of $\left.g_{T, t}\right)$. Andrews (1993), Sowell (1996), and Li and Müller (2009) discussed primitive conditions for these convergences. Sowell (1996) proceeded to derive weighted average power maximizing tests of (30) as a function of $G$ (that is, he computed $\varphi_{S}^{*}$ in the notation of Theorem 1) and he denoted the resulting test evaluated at $G_{T}(\cdot), \hat{H}_{T}$, and $\hat{V}_{T}$ (that is, $\hat{\varphi}_{T}^{*}$ in the notation of Theorem 1), an "optimal" test for structural change.

Without further restrictions, however, such tests cannot claim to be efficient. As a simple example, consider the scalar model with $y_{T, t}=\beta+T^{-1 / 2} \theta(t / T)+$ $\varepsilon_{t}$, where $\varepsilon_{t}$ is i.i.d. with $P\left(\varepsilon_{t}=-1\right)=P\left(\varepsilon_{t}=1\right)=1 / 2$. This model is a standard time varying parameter GMM model with $g_{T, t}(\beta)=y_{T, t}-\beta$ and $\hat{\beta}_{T}=T^{-1} \sum_{t=1}^{T} y_{T, t}$ satisfying (31), yet in this model, the test $\varphi_{T}^{* *}$ that rejects whenever any one of $\left\{y_{T, t}-y_{T, t-1}\right\}_{t=1}^{T}$ is not $-2,0$, or 2 has level zero for any $T \geq 2$ and has asymptotic power equal to 1 against any local alternative.

Theorem 1 provides a sense in which the tests derived by Sowell (1996) are asymptotically optimal: they maximize asymptotic weighted average power among all tests that have correct asymptotic null rejection probability whenever (31) holds with $\theta=0$. More powerful tests that exploit specificities of the error distribution, such as $\varphi_{T}^{* *}$, do not lead to correct asymptotic null rejection probability for all stable models satisfying (31). 


\section{CONCLUSION}

This paper analyzes an alternative notion of asymptotic efficiency of tests. The starting point is the idea that in many models, natural statistics of interest converge weakly under reasonable primitive assumptions. An observed random element thus converges weakly to a limiting random element, whose distribution is different under the null hypothesis and local alternative. It is shown that if one restricts attention to the class of tests that remain asymptotically valid for all models that induce the weak convergence, then efficient tests in the original problem are simply given by efficient tests in the limiting problem (that is, with the limiting random element observed), evaluated at sample analogues. These efficient tests generically coincide with robustified versions of efficient tests that are derived as the limit of small sample efficient tests in canonical parametric versions of the model. The results of this paper thus provide an alternative and broader sense of asymptotic efficiency for many previously derived tests in econometrics.

It is a strong restriction to force tests to have correct asymptotic null rejection probability for all models that satisfy the weak convergence, because there are typically many such models. Some of these models one might be willing to rule out a priori, which reduces the appeal of the efficiency claim. At the same time, the setup allows for a general and easily applied efficiency claim under minimal additional regularity conditions. The approach of the paper and the traditional semiparametric derivations are thus best seen as complements.

\section{APPENDIX}

PROOF OF THEOREM 1: (i) Since $\varphi_{S}^{*}$ is $\mu_{P}$ almost everywhere continuous, it is also $P(\theta)$ almost everywhere continuous for any $\theta \in \Theta$ by assumption about the family of measures $P(\theta)$, and by the continuous mapping theorem (CMT), $P_{T}(m, \theta) \rightsquigarrow P(\theta)$ implies

$$
\int \varphi_{S}^{*} d P_{T}(m, \theta) \rightarrow \int \varphi_{S}^{*} d P(\theta)
$$

for all $\theta \in \Theta$. Thus (8) and (10) hold for $\hat{\varphi}_{T}^{*}$. Similarly, also the $S \mapsto \mathbb{R}$ mapping $\left(\varphi_{S}^{*}-\alpha\right) f_{S}$ is $\mu_{P}$ almost everywhere continuous, so (11) follows from the CMT too. The last claim follows from (32) and dominated convergence, since $0 \leq$ $\varphi_{S}^{*} \leq 1$.

(ii) Heuristically, the proof follows the basic logic of constructing a tilted probability measure from a given alternative model described in the main text, but it addresses the difficulty that (a) $L^{i}$ is not necessarily bounded and (b) the testing problem is more general than a simple null against a simple alternative, and tests are further constrained by similarity and unbiasedness conditions. 
The proof consists of three steps. Consider first complication (a). Note that in the limit where $X \sim P(\theta)$, any change of measure from $P\left(\theta_{1}\right)$ to $P\left(\theta_{0}\right)$ using $L=d P\left(\theta_{0}\right) / d P\left(\theta_{1}\right), \int_{A} d P\left(\theta_{0}\right)=\int_{A} \frac{1}{L} d P\left(\theta_{1}\right)=\int_{A} \frac{1}{L} L d P\left(\theta_{0}\right)$ for all $A \in \mathfrak{B}(S)$, is well defined, even if $L^{i}$ is unbounded. The idea of the proof is thus to perform the tilting "in the limit" (Step 2) and ensure that it has the desired weak convergence implications for $X_{T}$ by constructing a probability space in which $X_{T}=h\left(Y_{T}\right) \rightarrow X$ almost surely (Step 1). Such almost sure representations of weakly converging random elements are known to exist in a complete and separable space. Because $X_{T}$ is a function of $Y_{T}$ and the set of models $\mathcal{M}$ is defined relative to the distribution of $Y_{T}$, we extend $h_{T}$ in a way that preserves the information about the distribution of $Y_{T}$. The tilting of the limiting measure in Step 2 then also implies the appropriate change of measure for $Y_{T}=h_{T}\left(X_{T}\right)$, as demonstrated in Step 3.

Due to complication (b), we cannot directly apply the Neyman-Pearson lemma to learn about the efficiency of a candidate test $\varphi_{T}$ for distinguishing between the original alternative model and the tilted model. Rather, we compare $\varphi_{T}$ with $\varphi_{S}^{*}$ directly in the limit in Step 2 by making its (best) asymptotic performance under the alternative part of the almost surely converging construction in Step 1. This is possible, because (randomized) tests take on values in the unit interval, so that any sequence of $\varphi_{T}$ has a subsequence that converges weakly by Prohorov's theorem. Because the tilting in the limit is a function of $X$ only, $X$ is a sufficient statistic, so that $\varphi_{S}^{*}(X)$ is an overall best test for learning about the tilting: see Step 2. Finally, as $\varphi_{T}$ is known to satisfy (8), (10), and (11), its weak limit is a level $\alpha$ test, and satisfies (5) and (6) under the tilted probability measures, so that it cannot be better than $\varphi_{S}^{*}$ : see Step 3 .

Step 1 . Pick any $m \in \mathcal{M}$. Define

$$
\bar{F}_{T}=\int F_{T}(m, \theta) d w(\theta), \quad \bar{P}_{T}=\int P_{T}(m, \theta) d w(\theta),
$$

and

$$
\bar{P}=\int P(\theta) d w(\theta) .
$$

For any bounded and continuous function $\vartheta: S \mapsto \mathbb{R}, \int \vartheta d P_{T}(m, \theta) \rightarrow$ $\int \vartheta d P(\theta)$ for all $\theta \in \Theta_{1}$ by the CMT, so that by dominated convergence, also $\int \vartheta d \bar{P}_{T} \rightarrow \int \vartheta d \bar{P}$. Thus, $h_{T} \bar{F}_{T}=\bar{P}_{T} \rightsquigarrow \bar{P}$. Let $D_{[0,1]}^{n}$ be the space of $n$-valued cadlag functions on the unit interval, equipped with the Billingsley (1968) metric, and define the mapping $\chi_{T}: \mathbb{R}^{n T} \mapsto D_{[0,1]}^{n}$ as $\left\{y_{t}\right\}_{t=1}^{T} \mapsto T^{-1} \Phi_{Z}\left(y_{\llcorner\cdot T]}\right)$, where $\Phi_{Z}$ is the cumulative distribution function of a standard normal applied element by element. Note that $\chi_{T}$ is injective and denote by $\chi_{T}^{-1}$ a $D_{[0,1]}^{n} \mapsto \mathbb{R}^{n T}$ function such that $\chi_{T}^{-1}\left(\chi_{T}(y)\right)=y$ for all $y \in \mathbb{R}^{n T}$. Since $\sup _{s \in[0,1]}\left\|\chi_{T}(s)\right\| \leq$ $1 / T \rightarrow 0$, the probability measures $\left(h_{T}, \chi_{T}\right) \bar{F}_{T}$ on the complete and separable space $S \times D_{[0,1]}^{n}$ converge weakly to the product measure $\bar{P} \times \delta_{0}$, where 
$\delta_{0}$ puts all mass at the zero function in $D_{[0,1]}^{n}$. Let $T_{1} \rightarrow \infty$ be any subsequence of $T$ such that $\lim _{T_{1} \rightarrow \infty} \int \varphi_{T_{1}} d \bar{F}_{T_{1}}=\limsup _{T \rightarrow \infty} \int \varphi_{T} d \bar{F}_{T}$. Since the probability measures $\left(h_{T_{1}}, \chi_{T_{1}}, \varphi_{T_{1}}\right) \bar{F}_{T_{1}}$ on the complete and separable space $S \times D_{[0,1]}^{n} \times[0,1]$ are uniformly tight, by Prohorov's theorem (see, for instance, Theorem 36 of Pollard (2002, p. 185)), there exists a subsequence $T_{2}$ of $T_{1}$ such that $\left(h_{T_{2}}, \chi_{T_{2}}, \varphi_{T_{2}}\right) \bar{F}_{T_{2}} \rightsquigarrow \bar{\nu}$ as $T_{2} \rightarrow \infty$, where $\left(\pi_{X}, \pi_{Y}\right) \bar{\nu}=\bar{P} \times \delta_{0}$, and $\pi_{X}, \pi_{Y}$, and $\pi_{\varphi}$ are the projections of $S \times D_{[0,1]}^{n} \times[0,1]$ on $S, D_{[0,1]}^{n}$, and $[0,1]$, respectively. For notational convenience, write $T$ for $T_{2}$ in the following. By Theorem 11.7.2 of Dudley (2002), there exists a probability space $\left(\Omega^{*}, \mathcal{F}^{*}, P^{*}\right)$ and functions $\eta_{T}: \Omega^{*} \mapsto S \times D_{[0,1]}^{n} \times[0,1]$ such that $\eta_{T} P^{*}=\left(h_{T}, \chi_{T}, \varphi_{T}\right) \bar{F}_{T}, \eta_{0} P^{*}=\bar{\nu}$, and $\eta_{T}\left(\omega^{*}\right) \rightarrow \eta_{0}\left(\omega^{*}\right)$ for $P^{*}$ almost all $\omega^{*} \in \Omega^{*}$, and by Theorem 11.7.3 of Dudley (2002), we may assume $\Omega^{*}$ to be complete and separable. In this construction, note that for $P^{*}$ almost all $\omega^{*}$, $h_{T} \circ \chi_{T}^{-1} \circ \pi_{Y} \circ \eta_{T}\left(\omega^{*}\right)=\pi_{X} \circ \eta_{T}\left(\omega^{*}\right)$ and $\varphi_{T} \circ \chi_{T}^{-1} \circ \pi_{Y} \circ \eta_{T}\left(\omega^{*}\right)=\pi_{\varphi} \circ \eta_{T}\left(\omega^{*}\right)$. Furthermore, $\left(\pi_{X} \circ \eta_{T}\right) P^{*}=\bar{P}_{T},\left(\chi_{T}^{-1} \circ \pi_{Y} \circ \eta_{T}\right) P^{*}=\bar{F}_{T}$, and $\int \varphi_{T} d \bar{F}_{T}=$ $\int\left(\pi_{\varphi} \circ \eta_{T}\right) d P^{*} \rightarrow \int\left(\pi_{\varphi} \circ \eta_{0}\right) d P^{*}$, where the convergence follows from the dominated convergence theorem, since $\pi_{\varphi} \circ \eta_{T}\left(\omega^{*}\right) \in[0,1]$ for all $\omega^{*} \in \Omega^{*}$. We need to show that $\int\left(\pi_{\varphi} \circ \eta_{0}\right) d P^{*} \leq \int \varphi_{S}^{*} d \bar{P}$.

Step 2 . Note that $P(\theta)$ is absolutely continuous with respect to $\bar{P}$ for any $\theta \in \Theta$, and denote by $L(\theta)$ the Radon-Nikodym derivative of $P(\theta)$ with respect to $\bar{P}$, so that for all $A \in \mathfrak{B}(S), \int_{A} d P(\theta)=\int_{A} L(\theta) d \bar{P}$ (existence of $L$ is ensured by the Radon-Nikodym theorem; see, for instance, Pollard (2002, p. 56)). Define $Q^{*}(\theta)$ to be the probability measure on $\mathcal{F}^{*}$, indexed by $\theta \in \Theta$, as $\int_{A} d Q^{*}(\theta)=\int_{A}\left(L(\theta) \circ \pi_{X} \circ \eta_{0}\right) d P^{*}$ for all $A \in \mathcal{F}^{*}$. Note that $Q^{*}(\theta)$ is a probability kernel. By construction, $\left(\pi_{X} \circ \eta_{0}\right) Q^{*}(\theta)=P(\theta)$, since for all $A \in \mathfrak{B}(S), \int_{A}\left(\pi_{X} \circ \eta_{0}\right) d Q^{*}(\theta)=\int_{A} L(\theta) d \bar{P}=\int_{A} d P(\theta)$. Consider the hypothesis test

$$
H_{0}^{*}: \omega^{*} \sim Q^{*}(\theta), \quad \theta \in \Theta_{0} \quad \text { against } \quad H_{1}^{*}: \omega^{*} \sim Q^{*}(\theta), \quad \theta \in \Theta_{1} .
$$

Because the Radon-Nikodym derivative between $Q^{*}\left(\theta_{1}\right)$ and $Q^{*}\left(\theta_{2}\right)$ is given by $\left(L\left(\theta_{1}\right) / L\left(\theta_{2}\right)\right) \circ \pi_{X} \circ \eta_{0}$, the statistic $\pi_{X} \circ \eta_{0}: \Omega^{*} \mapsto S$ is sufficient for $\theta$ by the factorization theorem (see, for instance, Theorem 2.21 of Schervish (1995)). Thus, for any test $\varphi_{\Omega}: \Omega^{*} \mapsto[0,1]$, one can define a corresponding test $\varphi_{S}: S \mapsto[0,1]$ via $\varphi_{S}(x)=E\left[\varphi_{\Omega} \mid\left(\pi_{X} \circ \eta_{0}\right)\left(\omega^{*}\right)=x\right]$, which satisfies $\int \varphi_{\Omega} d Q^{*}(\theta)=\int\left(\varphi_{S} \circ \pi_{X} \circ \eta_{0}\right) d Q^{*}(\theta)=\int \varphi_{S} d P(\theta)$ for all $\theta \in \Theta$ (cf. Theorem 3.18 of Schervish (1995)), and also $\int\left(f_{S} \circ \pi_{X} \circ \eta_{0}\right)\left(\varphi_{\Omega}-\alpha\right) d Q^{*}(\theta)=$ $\int f_{S}\left(\varphi_{S}-\alpha\right) d P(\theta)$ for any $f_{S} \in \mathcal{F}_{S}$. Since the level $\alpha$ test $\varphi_{S}^{*}: S \mapsto[0,1]$ of $H_{0}: X \sim P(\theta), \theta \in \Theta_{0}$, against $H_{1}: X \sim P(\theta), \theta \in \Theta_{1}$, maximizes weighted average power subject to (5) and (6), the level $\alpha$ test $\varphi_{S}^{*} \circ \pi_{X} \circ \eta_{0}: \Omega^{*} \mapsto[0,1]$ of (33) maximizes weighted average power $\iint \varphi_{\Omega} d Q^{*}(\theta) d w(\theta)$ among all level 
$\alpha$ tests $\varphi_{\Omega}: \Omega^{*} \mapsto[0,1]$ of (33) that satisfy

$$
\begin{aligned}
& \int \varphi_{\Omega} d Q^{*}(\theta) \geq \pi_{0}(\theta) \text { for all } \theta \in \Theta_{1}, \\
& \int\left(f_{S} \circ \pi_{X} \circ \eta_{0}\right)\left(\varphi_{\Omega}-\alpha\right) d Q^{*}(\theta)=0 \text { for all } \theta \in \bar{\Theta}_{0} \text { and } f_{S} \in \mathcal{F}_{S},
\end{aligned}
$$

and it achieves the same weighted average power

$$
\begin{aligned}
\iint\left(\varphi_{S}^{*} \circ \pi_{X} \circ \eta_{0}\right) d Q^{*}(\theta) d w(\theta) & =\iint \varphi_{S}^{*} d P(\theta) d w(\theta) \\
& =\int \varphi_{S}^{*} d \bar{P} .
\end{aligned}
$$

Step 3. Now define the sequence of measures $G_{T}(\theta)$ on $\mathfrak{B}\left(\mathbb{R}^{n T}\right)$, indexed by $\theta \in \Theta$, via $G_{T}(\theta)=\left(\chi_{T}^{-1} \circ \pi_{Y} \circ \eta_{T}\right) Q^{*}(\theta)$, which induce the measures $h_{T} G_{T}(\theta)=\left(h_{T} \circ \chi_{T}^{-1} \circ \pi_{Y} \circ \eta_{T}\right) Q^{*}(\theta)=\left(\pi_{X} \circ \eta_{T}\right) Q^{*}(\theta)$ on $\mathfrak{B}(S)$. By construction of $\eta_{T}$ and absolute continuity of $Q^{*}(\theta)$ with respect to $P^{*}$, we have $\eta_{T}\left(\omega^{*}\right) \rightarrow \eta_{0}\left(\omega^{*}\right)$ for $Q^{*}(\theta)$ almost all $\omega^{*}$, and since almost sure convergence implies weak convergence, $h_{T} G_{T}(\theta) \rightsquigarrow\left(\pi_{X} \circ \eta_{0}\right) Q^{*}(\theta)=P(\theta)$ pointwise in $\theta \in \Theta . G_{T}(\theta)$ thus defines a model $m_{0} \in \mathcal{M}$. Since $\varphi_{T}$ satisfies (8), (10), and (11), we have $\lim \sup _{T \rightarrow \infty} \int \varphi_{T} d G_{T}(\theta) \leq \alpha$ for all $\theta \in \Theta_{0}$, $\liminf _{T \rightarrow \infty} \int \varphi_{T} d G_{T}(\theta) \geq \pi_{0}(\theta)$ for all $\theta \in \Theta_{1}$, and $\lim _{T \rightarrow \infty} \int\left(\varphi_{T}-\alpha\right)\left(f_{S} \circ\right.$ $\left.h_{T}\right) d G_{T}(\theta)=0$ for all $\theta \in \bar{\Theta}_{0}$ and $f_{S} \in \mathcal{F}_{S}$. By the dominated convergence theorem and the construction of $G_{T}, \int \varphi_{T} d G_{T}(\theta)=\int\left(\varphi_{T} \circ \chi_{T}^{-1} \circ\right.$ $\left.\pi_{Y} \circ \eta_{T}\right) d Q^{*}(\theta)=\int\left(\pi_{\varphi} \circ \eta_{T}\right) d Q^{*}(\theta) \rightarrow \int\left(\pi_{\varphi} \circ \eta_{0}\right) d Q^{*}(\theta)$ for all $\theta \in \Theta$ and $\int\left(\varphi_{T}-\alpha\right)\left(f_{S} \circ h_{T}\right) d G_{T}(\theta)=\int\left(\left(\pi_{\varphi} \circ \eta_{T}\right)-\alpha\right)\left(f_{S} \circ \pi_{X} \circ \eta_{T}\right) d Q^{*}(\theta) \rightarrow$ $\int\left(\left(\pi_{\varphi} \circ \eta_{0}\right)-\alpha\right)\left(f_{S} \circ \pi_{X} \circ \eta_{0}\right) d Q^{*}(\theta)$ for all $\theta \in \bar{\Theta}_{0}$ and $f_{S} \in \mathcal{F}_{S}$. We conclude that the test $\pi_{\varphi} \circ \eta_{0}: \Omega^{*} \mapsto[0,1]$ of (33) is of level $\alpha$ and satisfies (34) and (35). Its weighted average power $\iint\left(\pi_{\varphi} \circ \eta_{0}\right) d Q^{*}(\theta) d w(\theta)$ is, therefore, smaller than or equal to the weighted average power $\iint \varphi_{S}^{*} d P(\theta) d w(\theta)$ of the test $\varphi_{S}^{*} \circ \pi_{X} \circ \eta_{0}: \Omega^{*} \mapsto[0,1]$. The result now follows from noting that $\iint\left(\pi_{\varphi} \circ \eta_{0}\right) d Q^{*}(\theta) d w(\theta)=\iint\left(L(\theta) \circ \pi_{X} \circ \eta_{0}\right)\left(\pi_{\varphi} \circ \eta_{0}\right) d P^{*} d w(\theta)=$ $\int\left(\pi_{\varphi} \circ \eta_{0}\right) d P^{*}$, since $\int L(\theta) d w(\theta)=1 \bar{P}$ almost surely and the change of the order of integration is allowed by Fubini's theorem.

Q.E.D.

Proof of Theorem 2: For notational convenience, write $F_{0, T}=F_{T}(m, \theta)$, $P_{T}=h_{T} F_{0, T}, P_{0, T}^{\phi}=\left(h_{T} \circ \phi_{T}\right) F_{0, T}, P_{0}=P(\theta)$, and $P_{0}^{\phi}=\tilde{\phi} P_{0}$. Proceed in analogy to the proof of Theorem 1(ii) to argue for the existence of a probability space $\left(\Omega^{*}, \mathcal{F}^{*}, P^{*}\right)$ with complete and separable $\Omega^{*}$ and functions $\eta_{T}: \Omega^{*} \mapsto$ $S \times D_{[0,1]}^{n}$ such that $\eta_{T} P^{*}=\left(h_{T} \circ \phi_{T}, \chi_{T}\right) F_{0, T}, \eta_{0} P^{*}=P_{0}^{\phi} \times \delta_{0}$, where the probability measure $\delta_{0}$ puts all mass on the zero function $D_{[0,1]}^{n} \mapsto \mathbb{R}$, and $\eta_{T}\left(\omega^{*}\right) \rightarrow \eta_{0}\left(\omega^{*}\right)$ for $P^{*}$ almost all $\omega^{*} \in \Omega^{*}$. In particular, $\left(\pi_{X} \circ \eta_{T}\right) P^{*}=P_{0, T}^{\phi}$ 
and $\left(\chi_{T}^{-1} \circ \pi_{Y} \circ \eta_{T}\right) P^{*}=F_{0, T}$, where $\pi_{X}$ and $\pi_{Y}$ are the projections of $S \times D_{[0,1]}^{n}$ on $S$ and $D_{[0,1]}^{n}$, respectively. Also, for $P^{*}$ almost all $\omega^{*}, h_{T} \circ \phi_{T} \circ \chi_{T}^{-1} \circ \pi_{Y} \circ$ $\eta_{T}\left(\omega^{*}\right)=\pi_{X} \circ \eta_{T}\left(\omega^{*}\right)$.

Let $\nu$ be the probability measure on $\mathfrak{B}(R \times S)$ induced by $(\tilde{\rho}, \tilde{\phi}): S \mapsto R \times S$ under $P_{0}$, that is, $\nu=(\tilde{\rho}, \tilde{\phi}) P_{0}$. Since $x=\tilde{g}(\tilde{\rho}(x), \tilde{\phi}(x))$ for all $x \in S, P_{0}=\tilde{g} \nu$. By Proposition 10.2.8 of Dudley (2002) there exists a probability kernel $\nu_{x}$ from $(S, \mathfrak{B}(S))$ to $(R, \mathfrak{B}(R))$ such that for each $A \in \mathfrak{B}(S)$ and $B \in \mathfrak{B}(R), \nu(A \times$ $B)=\int_{A} \nu_{x}(B) d P_{0}^{\phi}(x)$. Note that the $\Omega^{*} \times \mathfrak{B}(R) \mapsto[0,1]$ mapping defined via $\left(\omega^{*}, B\right) \mapsto \nu_{\pi_{X \circ \eta_{0}\left(\omega^{*}\right)}}(B)$ is a probability kernel from $\left(\Omega^{*}, \mathcal{F}^{*}\right)$ to $(R, \mathfrak{B}(R))$. We can thus construct the probability measure $\mu^{*}$ on $\left(\Omega^{*} \times R\right),\left(\mathcal{F}^{*} \otimes \mathfrak{B}(R)\right)$ via $\mu^{*}(C \times B)=\int_{C} \nu_{\pi_{X} \circ \eta_{0}\left(\omega^{*}\right)}(B) d P^{*}\left(\omega^{*}\right)$, and by construction, the mapping $\left(\omega^{*}, r\right) \mapsto \tilde{g}\left(r, \pi_{X} \circ \eta_{0}\left(\omega^{*}\right)\right)$ induces the measure $P_{0}$ under $\mu^{*}$.

Let $\xi_{T}\left(\omega^{*}, r\right)=h_{T}\left(g_{T}\left(r, \phi_{T} \circ \chi_{T}^{-1} \circ \pi_{Y} \circ \eta_{T}\left(\omega^{*}\right)\right)\right)$ and $\tilde{\xi}_{T}\left(\omega^{*}, r\right)=\tilde{g}\left(r, \pi_{X} \circ\right.$ $\left.\eta_{T}\left(\omega^{*}\right)\right)$. Let $\tilde{d}_{S} \leq 1$ be a metric equivalent to $d_{S}$. By assumption (iv), $\tilde{d}_{S}\left(\xi_{T}\left(\omega^{*}, r\right), \tilde{\xi}_{T}\left(\omega^{*}, r\right)\right)$ converges to zero in $P^{*}$ probability for any fixed $r \in R$. Thus, it also converges to zero in $\mu^{*}$ probability by dominated convergence. Furthermore, $\tilde{\xi}_{T}\left(\omega^{*}, r\right) \rightarrow \tilde{g}\left(r, \pi_{X} \circ \eta_{0}\left(\omega^{*}\right)\right)$ for $\mu^{*}$ almost all $\left(\omega^{*}, r\right)$ by the continuity of $\tilde{g}$. Since convergence in probability, and also almost sure convergence, imply weak convergence, the measures $G_{T}$ on $\mathfrak{B}\left(\mathbb{R}^{n T}\right)$ induced by the mapping $\left(\omega^{*}, r\right) \mapsto g_{T}\left(r, \phi_{T} \circ \chi_{T}^{-1} \circ \pi_{Y} \circ \eta_{T}\left(\omega^{*}\right)\right)$ under $\mu^{*}$ satisfy $h_{T} G_{T} \rightsquigarrow P_{0}$. Finally, from $\phi_{T} \circ g_{T}\left(r, \phi_{T}(y)\right)=\phi_{T}(y)$ for all $r \in R$ and $y \in \mathbb{R}^{n T}$, it follows that $\phi_{T} G_{T}=\left(\phi_{T} \circ \chi_{T}^{-1} \circ \pi_{Y} \circ \eta_{T}\right) P^{*}=\phi_{T} F_{0, T}$.

Q.E.D.

Proof OF THEOREM 3: The equality follows as in the proof of Theorem 1(i).

For the inequality, write $P_{0}=P\left(\theta_{0}\right), \bar{P}=\bar{L} P_{0}, F_{0, T}=F_{T}\left(m_{0}, \theta_{0}\right), P_{0, T}=$ $h_{T} F_{0, T}, \bar{F}_{T}=\int F_{T}\left(m_{1}, \theta\right) d w(\theta)$, and $\bar{P}_{T}=h_{T} \bar{F}_{T}$, so that $P_{0, T} \rightsquigarrow P_{0}$ and $\bar{P}_{T} \rightsquigarrow \bar{P}$ by assumption. Pick $1 / 2>\epsilon>0$ such that $P_{0}(\bar{L}=\epsilon)=0$ and define $B_{\epsilon}=$ $\{x \in S: \bar{L}(x)>\epsilon\}$. Note that $\int_{S \backslash B_{\epsilon}} d \bar{P}=\int_{S \backslash B_{\epsilon}} \bar{L} d P_{0} \leq \epsilon \int_{S \backslash B_{\epsilon}} d P_{0} \leq \epsilon$, so that $\int_{B_{\epsilon}} d \bar{P} \geq 1-\epsilon$. The assumption about $\bar{L}$ in the statement of the theorem also implies the existence of an open set $\bar{B}_{\epsilon}$ such that $\int_{\bar{B}_{\epsilon}} d \bar{P}>1-\epsilon$ and $\bar{L}: \bar{B}_{\epsilon} \mapsto \mathbb{R}$ is Lipschitz, since $\bar{P}$ is absolutely continuous with respect to $P_{0}$ (cf. Example 3 of Pollard (2002, p. 55)). With $B=B_{\epsilon} \cap \bar{B}_{\epsilon}, \bar{L}^{i}: B \mapsto \mathbb{R}$ with $\bar{L}^{i}=1 / \bar{L}$ is thus bounded and Lipschitz. Furthermore, since $S \backslash B$ is closed, there exists a Lipschitz function $\ell: S \mapsto[0,1]$ that is zero on $S \backslash B$ and for which $\int \ell d \bar{P} \geq 1-3 \epsilon$ (see Pollard (2002, pp. 172-173) for an explicit construction). For future reference, define $\mathcal{B}$ and $\mathcal{B}_{\epsilon}$ as the indicator functions of $B$ and $B_{\epsilon}$, respectively, and note that $\mathcal{B} \ell=\mathcal{B}_{\epsilon} \ell=\ell$. Define the scalar sequence

$$
\kappa_{T}=\int\left(\ell \circ h_{T}\right) d F_{0, T} / \int\left(\ell \circ h_{T}\right)\left(\bar{L}^{i} \circ h_{T}\right) d \bar{F}_{T}
$$




$$
=\int \ell d P_{0, T} / \int \ell \bar{L}^{i} d \bar{P}_{T}
$$

and note that $\kappa_{T} \rightarrow 1$ because $\int \ell d P_{0, T} \rightarrow \int \ell d P_{0}$ and $\int \ell \bar{L}^{i} d \bar{P}_{T} \rightarrow \int \ell \bar{L}^{i} d \bar{P}=$ $\int \mathcal{B} \ell \bar{L}^{i} \bar{L} d P_{0}=\int \ell d P_{0}$ by the continuous mapping theorem. Further define the probability distribution $G_{T}$ on $\mathfrak{B}\left(\mathbb{R}^{n T}\right)$ via

$$
\int_{A} d G_{T}=\kappa_{T} \int_{A}\left(\ell \circ h_{T}\right)\left(\bar{L}^{i} \circ h_{T}\right) d \bar{F}_{T}+\int_{A}\left((1-\ell) \circ h_{T}\right) d F_{0, T}
$$

for any $A \in \mathfrak{B}\left(\mathbb{R}^{n T}\right)$. Then by construction, $Q_{T}=h_{T} G_{T}$, where $Q_{T}$ satisfies

$$
\int_{A} d Q_{T}=\kappa_{T} \int_{A} \ell \bar{L}^{i} d \bar{P}_{T}+\int_{A}(1-\ell) d P_{0, T}
$$

for any $A \in \mathfrak{B}(S)$. Now

$$
\begin{aligned}
\Delta_{B L}\left(Q_{T}, P_{0}\right)= & \sup _{\|\vartheta\|_{B L} \leq 1}\left|\int \vartheta\left(d Q_{T}-d P_{0}\right)\right| \\
\leq & \sup _{\|\vartheta\|_{B L} \leq 1}\left|\int \vartheta \ell \bar{L}^{i}\left(\kappa_{T} d \bar{P}_{T}-d \bar{P}\right)\right| \\
& +\sup _{\|\vartheta\|_{B L} \leq 1}\left|\int \vartheta(1-\ell)\left(d P_{0, T}-d P_{0}\right)\right| \\
\leq & \left\|\ell \bar{L}^{i}\right\|_{B L}\left(\Delta_{B L}\left(\bar{P}_{T}, \bar{P}\right)+\left|\kappa_{T}-1\right|\right) \\
& +\|1-\ell\|_{B L} \Delta_{B L}\left(P_{0, T}, P_{0}\right),
\end{aligned}
$$

where the manipulations after the first inequality use $\int \vartheta \ell d P_{0}=\int \vartheta \ell \bar{L}^{i} d \bar{P}$ and the second inequality exploits that $\|\cdot\|_{B L}$ is a submultiplicative norm on the set of bounded Lipschitz functions $S \mapsto \mathbb{R}$ (cf. Proposition 11.2.1 of Dudley (2002)). Also

$$
\begin{aligned}
\left|\kappa_{T}-1\right| & =\left|\frac{\int \ell d P_{0, T}}{\int \ell \bar{L}^{i} d \bar{P}_{T}}-\frac{\int \ell d P_{0}}{\int \ell \bar{L}^{i} d \bar{P}}\right| \\
& \leq \frac{\left\|\ell \bar{L}^{i}\right\|_{B L} \Delta_{B L}\left(\bar{P}_{T}, \bar{P}\right)+\|\ell\|_{B L} \Delta_{B L}\left(P_{0, T}, P_{0}\right)}{\int \ell \bar{L}^{i} d \bar{P}_{T}} .
\end{aligned}
$$

Thus, $\lim _{T \rightarrow \infty} \Delta_{B L}\left(Q_{T}, P_{0}\right) / \delta_{T}=0$, so that (18) implies

$$
\limsup _{T \rightarrow \infty} \int \varphi_{T} d G_{T} \leq \alpha .
$$


Now define the probability measures $\tilde{F}_{T}$ on $\mathfrak{B}\left(\mathbb{R}^{n T}\right)$ via

$$
\begin{aligned}
\int_{A} d \tilde{F}_{T} & =\tilde{\kappa}_{T} \int_{A}\left(\mathcal{B}_{\epsilon} \circ h_{T}\right)\left(\bar{L} \circ h_{T}\right) d G_{T} \\
& =\tilde{\kappa}_{T} \kappa_{T} \int_{A}\left(\ell \circ h_{T}\right) d \bar{F}_{T}+\tilde{\kappa}_{T} \int_{A}\left(\mathcal{B}_{\epsilon}(1-\ell) \bar{L} \circ h_{T}\right) d F_{0, T}
\end{aligned}
$$

for any $A \in \mathfrak{B}\left(\mathbb{R}^{n T}\right)$, where $\tilde{\kappa}_{T}=1 /\left(\kappa_{T} \int\left(\ell \circ h_{T}\right) d \bar{F}_{T}+\int\left(\mathcal{B}_{\epsilon}(1-\ell) \bar{L} \circ\right.\right.$ $\left.\left.h_{T}\right) d F_{0, T}\right) \rightarrow \tilde{\kappa}=1 / \int\left(\ell+\mathcal{B}_{\epsilon}-\mathcal{B}_{\epsilon} \ell\right) d \bar{P}=1 / \int \mathcal{B}_{\epsilon} d \bar{P}$ and $1 \leq 1 / \int \mathcal{B}_{\epsilon} d \bar{P} \leq$ $1+2 \epsilon$. By the Neyman-Pearson lemma, the best test of $\tilde{H}_{0}: Y_{T} \sim G_{T}$ against $\tilde{H}_{1}: Y_{T} \sim \tilde{F}_{T}$ thus rejects for large values of $\left(\mathcal{B}_{\epsilon} \bar{L}\right) \circ h_{T}$, that is, $\bar{L} \circ h_{T}$.

For any $T$, denote by $\tilde{\varphi}_{T}^{*}: \mathbb{R}^{n T} \mapsto[0,1]$ the test that rejects for large values of $\bar{L} \circ h_{T}$ of level $\int \tilde{\varphi}_{T}^{*} d G_{T}=\max \left(\int \varphi_{T} d G_{T}, \alpha\right)$, so that $\int\left(\tilde{\varphi}_{T}^{*}-\varphi_{T}\right) d \tilde{F}_{T} \geq 0$ for all $T$. By Le Cam's first lemma (Lemma 6.4 in van der Vaart (1998)), $\tilde{F}_{T}$ is contiguous to $G_{T}$, since under $G_{T}$, the Radon-Nikodym derivative $\tilde{\kappa}_{T}\left(\mathcal{B}_{\epsilon} \bar{L}\right) \circ$ $h_{T}$ converges weakly to the distribution $\tilde{\kappa} \mathcal{B}_{\epsilon} \bar{L} P_{0}$ by the continuous mapping theorem, and $\int \tilde{\kappa} \mathcal{B}_{\epsilon} \bar{L} d P_{0}=1$. Since both $\tilde{\varphi}_{T}^{*}$ and $\hat{\varphi}_{T}^{*}$ reject for large values of $\bar{L} \circ h_{T}$ and are of asymptotic level $\alpha$, we have $\int\left|\tilde{\varphi}_{T}^{*}-\hat{\varphi}_{T}^{*}\right| d G_{T} \rightarrow 0$, so that by contiguity, also $\int\left|\tilde{\varphi}_{T}^{*}-\hat{\varphi}_{T}^{*}\right| d \tilde{F}_{T} \rightarrow 0$. Thus $\lim \sup _{T \rightarrow \infty} \int\left(\varphi_{T}-\hat{\varphi}_{T}^{*}\right) d \tilde{F}_{T} \leq 0$. To complete the proof, note that the total variation distance between $\tilde{F}_{T}$ and $\bar{F}_{T}$ is bounded above by

$$
\begin{aligned}
& \int\left|1-\tilde{\kappa}_{T} \kappa_{T}\left(\ell \circ h_{T}\right)\right| d \bar{F}_{T} \\
& \quad \leq\left|1-\tilde{\kappa}_{T} \kappa_{T}\right|+\left|\tilde{\kappa}_{T} \kappa_{T} \int(1-\ell) d \bar{P}_{T}\right| \\
& \quad \rightarrow 1 / \int \mathcal{B}_{\epsilon} d \bar{P}-1+\left(1-\int \ell d \bar{P}\right) / \int \mathcal{B}_{\epsilon} d \bar{P} \leq 8 \epsilon
\end{aligned}
$$

so that $\limsup _{T \rightarrow \infty} \int\left(\varphi_{T}-\hat{\varphi}_{T}^{*}\right) d \bar{F}_{T} \leq 8 \epsilon$, and the result follows, since $1 / 2>$ $\epsilon>0$ can be chosen arbitrarily small.

Q.E.D.

\section{REFERENCES}

Anderson, T. W., AND H. RuBIN (1949): "Estimators of the Parameters of a Single Equation in a Complete Set of Stochastic Equations," The Annals of Mathematical Statistics, 21, 570-582. [424]

ANDREWS, D. W. K. (1993): "Tests for Parameter Instability and Structural Change With Unknown Change Point," Econometrica, 61, 821-856. [425]

ANDrEWs, D. W. K., AND W. Ploberger (1994): “Optimal Tests When a Nuisance Parameter Is Present Only Under the Alternative," Econometrica, 62, 1383-1414. [395]

Andrews, D. W. K., M. J. MoreIRA, AND J. H. StOCK (2006): “Optimal Two-Sided Invariant Similar Tests for Instrumental Variables Regression,” Econometrica, 74, 715-752. [396,399,423] 
(2008): "Efficient Two-Sided Nonsimilar Invariant Tests in IV Regression With Weak Instruments," Journal of Econometrics, 146, 241-254. [396]

Bickel, P. J., C. A. J. KLAASSEN, Y. Ritov, AND J. A. Wellner (1998): Efficient and Adaptive Estimation for Semiparametric Models. New York: Springer-Verlag. [398]

Billingsley, P. (1968): Convergence of Probability Measure. New York: Wiley. [400,427]

BREITUNG, J. (2002): “Nonparametric Tests for Unit Roots and Cointegration," Journal of Econometrics, 108, 343-363. [404]

CHOI, A., W. J. HALL, AND A. SCHICK (1996): "Asymptotically Uniformly Most Powerful Tests in Parametric and Semiparametric Models," The Annals of Statistics, 24, 841-861. [398]

DAVIDSON, J. (2002): "Establishing Conditions for the Functional Central Limit Theorem in Nonlinear and Semiparametric Time Series Processes," Journal of Econometrics, 106, 243-269. [404] - (2009): "When Is a Time-Series I(0)?" in The Methodology and Practice of Econometrics: A Festschrift in Honour of David F. Hendry, ed. by J. Castle and N. Shephard. Oxford: Oxford University Press, 322-342. [404]

DE Jong, R. M., AND J. DAVIDSON (2000): “The Functional Central Limit Theorem and Weak Convergence to Stochastic Integrals I: Weakly Dependent Processes," Econometric Theory, 16, 621-642. [421]

DiCKEY, D. A., AND W. A. Fuller (1979): "Distribution of the Estimators for Autoregressive Time Series With a Unit Root," Journal of the American Statistical Association, 74, 427-431. [406]

Dudley, R. M. (2002): Real Analysis and Probability. Cambridge, U.K.: Cambridge University Press. [418,419,428,430,431]

Dufour, J.-M. (1997): "Some Impossibility Theorems in Econometrics With Applications to Structural and Dynamic Models," Econometrica, 65, 1365-1387. [418]

Dufour, J.-M., AND M. L. KING (1991): "Optimal Invariant Tests for the Autocorrelation Coefficient in Linear Regressions With Stationary or Nonstationary AR(1) Errors," Journal of Econometrics, 47, 115-143. [408]

ELLIOTT, G. (1999): "Efficient Tests for a Unit Root When the Initial Observation Is Drawn From Its Unconditional Distribution,” International Economic Review, 40, 767-783. [395]

ElliotT, G., AND M. JANSSON (2003): “Testing for Unit Roots With Stationary Covariates,” Journal of Econometrics, 115, 75-89. [395,399,420,421]

ElliotT, G., AND U. K. MÜLleR (2006): "Efficient Tests for General Persistent Time Variation in Regression Coefficients," Review of Economic Studies, 73, 907-940. [395]

(2009): "Pre and Post Break Parameter Inference," Working Paper, Princeton University. [401]

Elliott, G., M. Jansson, And E. Pesavento (2005): “Optimal Power for Testing Potential Cointegrating Vectors With Known Parameters for Nonstationarity,” Journal of Business \& Economic Statistics, 23, 34-48. [395]

Elliott, G., T. J. RothenberG, AND J. H. Stock (1996): "Efficient Tests for an Autoregressive Unit Root,” Econometrica, 64, 813-836. [395]

HANSEN, B. E. (1990): "Convergence to a Stochastic Integrals for Dependent Heterogeneous Processes," Econometric Theory, 8, 489-500. [421]

IBRAGIMOV, R., AND U. K. MÜLLER (2010): "T-Statistic Based Correlation and Heterogeneity Robust Inference," Journal of Business \& Economic Statistics, 28, 453-468. [398]

JANSSON, M. (2005): "Point Optimal Tests of the Null Hypothesis of Cointegration," Journal of Econometrics, 124, 187-201. [395]

(2008): "Semiparametric Power Envelopes for Tests of the Unit Root Hypothesis," Econometrica, 76, 1103-1142. [396-398,408,409,412,413]

JANSSON, M., AND M. J. MOREIRA (2006): “Optimal Inference in Regression Models With Nearly Integrated Regressors,” Econometrica, 74, 681-714. [396,405]

Jeganathan, P. (1995): "Some Aspects of Asymptotic Theory With Applications to Time Series Models," Econometric Theory, 11, 818-887. [398] 
KING, M. L. (1988): "Towards a Theory of Point Optimal Testing," Econometric Reviews, 6, 169-218. [400,401]

LE CAM, L. (1986): Asymptotic Methods in Statistical Decision Theory. New York: Springer-Verlag. [398]

LeHmann, E. L. (1986): Testing Statistical Hypotheses (Second Ed.). New York: Wiley. [401,411, 415]

LI, H., AND U. K. MÜLLER (2009): "Valid Inference in Partially Unstable General Method of Moment Models," Review of Economic Studies, 76, 343-365. [425]

LiNG, S., AND M. MCALEER (2003): "On Adaptive Estimation in Nonstationary ARMA Models With GARCH Errors," The Annals of Statistics, 31, 642-674. [398]

MCLeish, D. L. (1974): "Dependent Central Limit Theorems and Invariance Principles," The Annals of Probability, 2, 620-628. [404]

MoreIRA, M. J. (2001): "Tests With Correct Size When Instruments Can Be Arbitrarily Weak," Working Paper, University of California, Berkeley. [424]

MÜLLER, U. K. (2007): “A Theory of Robust Long-Run Variance Estimation,” Journal of Econometrics, 141, 1331-1352. [404]

(2008): "The Impossibility of Consistent Discrimination Between $\mathrm{I}(0)$ and $\mathrm{I}(1)$ Processes," Econometric Theory, 24, 616-630. [404]

(2009): "Comment on: Unit Root Testing in Practice Dealing With Uncertainty Over the Trend and Initial Condition," Econometric Theory, 25, 643-648. [395]

MÜLLER, U. K., AND G. ElLIOTT (2003): “Tests for Unit Roots and the Initial Condition,” Econometrica, 71, 1269-1286. [395,416]

MÜLLER, U. K., AND M. W. WATSON (2008a): "Low-Frequency Robust Cointegration Testing," Working Paper, Princeton University. [398,401] $[398,404,414]$

NEWEY, W. K. (1990): “Semiparametric Efficiency Bounds,” Journal of Applied Econometrics, 5, 99-135. [398]

NyBlOM, J. (1989): “Testing for the Constancy of Parameters Over Time," Journal of the American Statistical Association, 84, 223-230. [395]

PhILliPS, P. C. B. (1988): "Weak Convergence of Sample Covariance Matrices to Stochastic Integrals via Martingale Approximations," Econometric Theory, 4, 528-533. [421]

PhILLIPS, P. C. B., AND V. SOLO (1992): "Asymptotics for Linear Processes," The Annals of Statistics, 20, 971-1001. [404]

POLLARD, D. (2001): "Contiguity,” Working Paper, Yale University. [408]

(2002): A User's Guide to Measure Theoretic Probability. Cambridge, U.K.: Cambridge University Press. [419,428,430]

PÖTSCHER, B. M. (2002): "Lower Risk Bounds and Properties of Confidence Sets for Ill-Posed Estimation Problems With Applications to Spectral Density and Persistence Estimation, Unit Roots, and Estimation of Long Memory Parameters," Econometrica, 70, 1035-1065. [418]

ROTHENBERG, T. J., AND J. H. STOCK (1997): "Inference in a Nearly Integrated Autoregressive Model With Nonnormal Innovations," Journal of Econometrics, 80, 269-286. [396,412]

SCHERVISH, M. J. (1995): Theory of Statistics. New York: Springer. [428]

ShiryaeV, A. N., AND V. G. SpokoInY (2000): Statistical Experiments and Decisions: Asymptotic Theory. Singapore: World Scientific Publishing. [398]

Sowell, F. (1996): "Optimal Tests for Parameter Instability in the Generalized Method of Moments Framework," Econometrica, 64, 1085-1107. [398,399,409,425]

SRIANANTHAKUmar, S., AND M. L. King (2006): "A New Approximate Point Optimal Test of a Composite Null Hypothesis," Journal of Econometrics, 130, 101-122. [401]

STAIGER, D., AND J. H. STOCK (1997): "Instrumental Variables Regression With Weak Instruments," Econometrica, 65, 557-586. [423]

STEIN, C. (1956): "Efficient Nonparametric Estimation and Testing," in Proceedings of the Third Berkeley Symposium on Mathematical Statistics and Probability, Vol. 1. Berkeley: University of California Press, 187-196. [398,411] 
Stock, J. H. (1994): "Deciding Between I(1) and I(0)," Journal of Econometrics, 63, 105-131. [404]

(1999): "A Class of Tests for Integration and Cointegration," in Cointegration, Causality and Forecasting: A Festschrift in Honour of Clive W. J. Granger, ed. by R. F. Engle and H. White. Oxford: Oxford University Press, 135-167. [397,410]

STOCK, J. H., AND M. W. WATSON (1996): "Confidence Sets in Regressions With Highly Serially Correlated Regressors," Working Paper, Harvard University. [396]

StOck, J. H., AND J. H. WRIGHT (2000): “GMM With Weak Identification,” Econometrica, 68, 1055-1096. [409]

VAN DER VAART, A. W. (1998): Asymptotic Statistics. Cambridge, U.K.: Cambridge University Press. [398,432]

VAN DER VAART, A. W., AND J. A. Wellner (1996): Weak Convergence and Empirical Processes With Applications to Statistics. New York: Springer. [409]

WhITE, H. (2001): Asymptotic Theory for Econometricians (Revised Ed.). San Diego: Academic Press. [404]

Wooldridge, J. M., AND H. White (1988): "Some Invariance Principles and Central Limit Theorems for Dependent Heterogeneous Processes,” Econometric Theory 4, 210-230. [404]

Dept. of Economics, Princeton University, Princeton, NJ 08544, U.S.A.; umueller@princeton.edu.

Manuscript received March, 2008; final revision received September, 2010. 